\title{
Sur la formation des prix dans l'économie du haut Moyen Âge *
}

Laurent Feller

$\mathrm{Au}$ début $\mathrm{du} \mathrm{XI}^{\mathrm{e}}$ siècle, le narrateur des Miracles de sainte Foy de Conques, Bernard d'Angers, rapporte qu'un commerçant de Conques se livrait à une manœuvre à ses yeux scandaleuse. Il achetait en effet toute la cire disponible dans les environs de la cité, acquérant ainsi une position de monopole de vente pour la fourniture des cierges, ce qui lui permettait d'en tirer un prix élevé. La sainte ne manqua pas de le punir cruellement ${ }^{1}$. L'action du commerçant supposait une certaine pratique du marché, une connaissance empirique des mécanismes de formation des prix et des scrupules assez limités quant à la moralité de l'action, au demeurant tout à fait licite. Cette anecdote pose la question de la connaissance et de la maîtrise par les agents du haut Moyen Âge et par les témoins qui en rapportent les actes, de mécanismes économiques passablement complexes et dont, a priori, on tendrait à les penser ignorants ou dont on voudrait croire qu'ils les ignoraient volontairement. Les processus par lesquels les prix se forment ainsi que les procédures permettant d'assigner une valeur aux choses que l'on échange semblent, pour beaucoup d'historiens des sociétés du haut Moyen Âge, ne pas faire sens et en parler relever de la gageure.

En Occident, en effet, entre $\mathrm{VI}^{\mathrm{e}}$ et $\mathrm{XI}^{\mathrm{e}}$ siècles, échanges marchands et échanges non marchands s'entrecroisent au point de se confondre pour l'observateur dans un seul et même ensemble dont les éléments ne peuvent être distingués les uns des autres. On a ainsi pu considérer, en ce sens, qu'il n'y avait pas d'économie médiévale, parce que la production, l'échange et la consommation n'étaient pas des réalités distinctes des rites sociaux qui les encadraient ${ }^{2}$. Tout se passe dès lors comme si les formes et les institutions sociales du haut

\footnotetext{
Je tiens à remercier Florence Weber et Jean-Pierre Devroey de leur relecture ainsi que de leurs critiques et suggestions. J'en ai tenu le plus grand compte possible.

1 - Luca Robertini (éd.), Liber Miraculorum Sancte Fidis, Spolète, Centro italiano di studi sull'alto Medioevo, 1994, liv. I, chap. 24, p. 125-127. Voir le commentaire du passage dans Giacomo TODESCHINI, Richesse franciscaine. De la pauvreté volontaire à la société de marché, Lagrasse, Verdier, [2005] 2008, p. 19. Laurent FELLER, «Introduction. Formes et fonctions de la richesse des élites au haut Moyen Âge », in J.-P. DEVROEY, L. FELLER et R. LE JAN (dir.) Les élites et la richesse au haut Moyen Âge, Turnhout, Brepols, 2010, p. 5-30.

2 - Voir, par exemple, DominiQue IognA-PRAT, « Préparer l'au-delà, gérer l'ici-bas : les élites ecclésiastiques, la richesse et l'économie du christianisme (perspectives de travail)», in J.-P.
} 
Moyen Âge étaient semblables à celles que l'on trouve, de l'autre côté du « Grand Partage », chez les peuples « premiers » qui forment l'objet d'étude des anthropologues ${ }^{3}$ : on présente désormais comme une vérité d'évidence que l'enchâssement de l'économique dans le social rend impossible, voire inutile, tout effort de clarification et de distinction. On a affaire à une structure dont tous les éléments interagissent les uns sur les autres, et c'est alors l'interaction qui fait sens et non l'évolution de tel ou tel objet pris séparément.

Il est pourtant nécessaire de tenter l'aventure. Cette question recouvre en effet celle des conditions de possibilité de la croissance et du développement et l'on ne peut se contenter de constater, sans rechercher de cause première, l'essor de la production agraire, l'urbanisation, la commercialisation de l'économie, le développement des activités artisanales et industrielles, etc. Je voudrais présenter, dans ces pages, les directions qui peuvent être empruntées, et qui commencent à l'être, pour aborder ces questions. Il est d'autant plus nécessaire de le faire que les analyses couramment présentées et la conception que l'on développe actuellement de l'enchâssement de l'économique dans le social (embeddedness) ont eu pour effet de délégitimer en partie le champ de l'économique pour l'histoire du Moyen Âge, celui-ci en venant à être une figure ou un aspect de l'histoire culturelle, seule légitime ou seule apte à rendre compte de l'intégralité des processus régissant la vie humaine ${ }^{4}$.

La recherche a déjà commencé : les travaux récents les plus novateurs, ceux de Chris Wickham et de Jean-Pierre Devroey montrent des réalités beaucoup plus ambiguës ${ }^{5}$. Les comportements à l'égard de la richesse, de sa production et de sa transmission peuvent aussi

DeVroey, L. Feller et R. LE JAN (dir.) Les élites et la richesse..., op. cit., p. 59-70 ; Alain GUERrEAU, L'avenir d'un passé incertain. Quelle histoire du Moyen Âge au XXI' siècle ?, Paris, Le Seuil, 2001.

3 - Karl POLANYI, La grande transformation. Aux origines politiques et économiques de notre temps, Paris, Gallimard, [1944] 1983; Voir à propos de l'utilisation de K. Polanyi par les médiévistes français, les remarques roboratives, quoique brèves de Matthieu ARNOUX «Vérité et question des marchés médiévaux », in A. HATCHUEL, O. FAVEREAU et F. AGGERI (dir.), L'activité marchande sans le marché, Paris, Mines-Paris Tech, 2010, p. 27-43, ici p. 27.

4 - Laurent FELLER, « Histoire du Moyen Âge et histoire économique ( $\mathrm{X}^{\mathrm{e}}-\mathrm{XV}^{\mathrm{e}}$ siècle) en France », in S. CAVACCIOCHI (dir.), Où va l'histoire économique? Méthodes et perspectives. XIII'-XVIII siècles (XLII settimana di Studi, Prato, 18-22 avril 2010), Prato 2011, p. .

5 - Chris WiCKham, Framing the early Middle Ages: Europe and the Mediterranean, 400-800, Oxford, Oxford University Press, 2005 ; Jean-Pierre Devroey, Puissants et misérables. Système social et monde paysan dans l'Europe des Francs, VI $I^{e} X^{e}$ siècles, Bruxelles, Académie royale de Belgique, 2006. 
être analysés en termes de comportements rationnels, que l'on parle en finalité ou en valeur : c'est dans cette direction que recherches et programmes s'orientent à présent ${ }^{6}$. Les interrogations doivent cependant être déplacées et les considérations relevant purement de la science économique doivent être pondérées par une réflexion sur la dimension éthique des décisions et des calculs faits par les uns et les autres. La thèse qui est à présent développée est que le calcul économique existe bien, et qu'il est présent à côté des comportements imposés par l'organisation sociale et familiale comme par les obligations religieuses et morales ${ }^{7}$. S'il faut, par exemple, redistribuer d'un côté, faire preuve de largesse, il faut également, de l'autre, transmettre le patrimoine afin de perpétuer la lignée ; il est moins évident, mais cela se produit tout de même, que les agents investissent, se soucient de construire des biens d'infrastructure, de réorganiser leurs patrimoines pour accroître leurs revenus, tirer de leur allocation de ressources le maximum de profit afin d'augmenter aussi ce dont ils peuvent disposer pour le donner, pour le transmettre ou pour le consommer ${ }^{8}$.

Une direction a été insuffisamment prise jusqu'à présent, celle de l'examen des normes éthiques imposées par l'Église dès le haut Moyen Âge et de leurs conséquences sur les comportements. De façon apparemment paradoxale, le christianisme semble privilégier la pauvreté mais il s'accommode fort bien de la richesse et des procédures nécessaires pour la produire et la transmettre. S'il n'existe pas au haut Moyen Âge de véritable doctrine économique ou, si l'on préfère, s'il n'existe pas de pensée formalisée portant sur la production, la consommation et l'échange, il existe en revanche des normes de comportement bien établies, construites dès le $\mathrm{VI}^{\mathrm{e}}$ siècle dans les plus élaborées des règles monastiques et qui guident l'action gestionnaire des moines durant tout le Moyen Âge. Elles nous disent quelque chose de la nature des comportements et des cadres à l'intérieur desquels ceux-ci se construisent.

Je me propose ici, après avoir brièvement rappelé les complexités auxquelles sont parvenues les analyses récentes, notamment en ce qui concerne le marché de la terre,

\footnotetext{
6 - Voir l'ouvrage collectif issu de l'un des colloques du programme « Les élites au Moyen Âge » :

J.-P. DeVroey, L. Feller et R. Le JAN (dir.) Les élites et la richesse..., op. cit.

7 - Max Weber, Économie et société, t. 1, Les catégories de la sociologie, Paris, Pocket, 1995, p. 55 sq. ; J.-P. DeVRoeY, Puissants et misérables..., op. cit., p. 585-613.

8 - Voir les publications issues du programme «Transferts patrimoniaux au haut Moyen Âge » et, en particulier, François Bougard, Cristina LA RocCA et Régine LE JAN (dir.), Sauver son âme et se perpétuer. Transmission du patrimoine et mémoire au haut Moyen Âge, Rome, École française de Rome, 2005.
} 
d'examiner quelques aspects du comportement à l'égard des prix, aussi bien d'un point de vue pragmatique que moral. Voici déjà longtemps que Giacomo Todeschini a montré combien le lexique chrétien était saturé de références à l'échange marchand et au prix des $\operatorname{choses}^{9}:$ le moins que l'on puisse faire est de s'interroger sur les incidences de cette présence de la figure de l'achat et de l'échange marchand dans la vie quotidienne. La façon dont les prix se forment et sont énoncés est de ce fait un objet légitime, voire nécessaire, dans la mesure où, par hypothèse, il existe une sphère de l'échange marchand même dans une société où l'économique est «enchâssé » dans le social. C'est la validité de cette hypothèse que je voudrais commencer à tester ici.

\section{Historiographie}

Concernant l'histoire économique de la période, nous sommes d'abord confrontés à un problème de chronologie générale et de périodisation dont il n'est pas inutile de rappeler brièvement les termes. Henri Pirenne a imposé, dans les années 1930, une chronologie particulière à laquelle, d'une manière ou d'une autre, l'érudition est encore attachée et dont elle dépend partiellement. Sa construction repose sur une prise de parti théorique simple : durant tout le Moyen Âge, et même après, les revenus que l'on peut attendre du capital mobilier sont plus importants que ceux du capital foncier ${ }^{10}$. La terre ne produit pas de quoi nourrir un marché qui serait lucratif et permettrait d'accumuler de nouvelles richesses. En revanche, le commerce, principalement celui des objets de luxe, est constamment d'un rapport élevé. Alors que structures foncières et insuffisances techniques se cumulent pour empêcher jusqu'au XI ${ }^{e}$ siècle tout essor de la production agraire, mettant l'économie rurale hors-jeu dans la mise en place de mécanismes de croissance, les oscillations du grand commerce international déterminent, par l'importance des valeurs en cause, l'apparition des conditions

9 - Giacomo TODESChInI, Il prezzo della salvezza. Lessici medievali del pensiero economico, Rome, NIS, 1994 ; Id., Richesse franciscaine..., op. cit. ; Valentina TONEATTO, «Élites et rationalité économique. Les lexiques de l'administration monastique du haut Moyen Âge », in J.-P. DEVROEY, L. FELlER et R. LE JAN (dir.) Les élites et la richesse..., op. cit. p. 71-99 ; Id., " Marchands et banquiers du Seigneur. Lexiques chrétiens de la richesse et de l'administration monastiques de la fin du IV ${ }^{\mathrm{e}}$ siècle au début du IX $\mathrm{X}^{\mathrm{e}}$ siècle », thèse, université Paris-Ouest-La Défense, 2009.

10 - Henri PIREnNe, Mahomet et Charlemagne, Paris/Bruxelles, Alcan/ $\mathrm{N}^{\text {elle }} \mathrm{S}^{\text {té }}$ d'édition, 1936. On trouvera les points d'ancrage méthodologiques de sa réflexion dans Id., Histoire économique de l'Occident médiéval, Paris, Desclée De Brouwer, 1951. 
de possibilité du développement de l'Occident. Cette prise de position s'articule sur une chronologie précise. Les structures d'échange du monde antique perdurent au-delà de la disparition de ses institutions politiques et sont encore efficientes au VII siècle, ainsi que l'établissent les diplômes mérovingiens attestant de la présence de produits de luxe en quantités importantes dans le port de Fos ${ }^{11}$. À partir du VII ${ }^{\mathrm{e}}$ siècle, en revanche, la conquête musulmane brise les liens entre Orient et Occident et le «grand commerce international » cesse d'exister: la période carolingienne est un moment de contraction économique et d'appauvrissement de l'Occident, puisque le moteur de la croissance fait brutalement défaut. La reprise ne se produit qu'à partir du $\mathrm{XI}^{\mathrm{e}}$ siècle, lorsque les conditions de la vie urbaine en Flandre et en Italie permettent le démarrage d'un commerce à grande échelle au départ de l'Europe et portant cette fois sur le drap, qui est à la fois un produit de luxe et un produit de première nécessité. Les grands défrichements accompagnent ce mouvement mais ne le provoquent pas. Récemment, ces thèses ont été d'une certaine manière revivifiées par les archéologues spécialisés dans les périodes mérovingienne et carolingienne ${ }^{12}$. On trouve en effet dans les villes de l'époque mérovingienne d'importants signes d'activité commerciale. Sur les mêmes sites, la période $\mathrm{VIII}^{\mathrm{e}}-\mathrm{IX}^{\mathrm{e}}$ siècle se caractérise par l'absence de fossiles indiquant l'existence d'échanges commerciaux et d'activités de production ; on les retrouve à nouveau à partir du $\mathrm{XI}^{\mathrm{e}}$ siècle. Joachim Henning a, pour expliquer cette absence, émis l'hypothèse selon laquelle ces activités auraient été déplacées vers les monastères dont les autorités politiques carolingiennes auraient voulu faire les lieux centraux de la production et de l'échange, contribuant à provoquer une crise générale de l'économie au $\mathrm{IX}^{\mathrm{e}}$ siècle, souffrant d'un excès d'administration de la part de souverains finalement peu compétents en ces matières ${ }^{13}$.

\footnotetext{
11 - H. PIRENNE, Mahomet et Charlemagne, op. cit., p. 69-61.

12 - Joachim HenNing, «Strong rulers - weak economy? Rome, the Carolingians and the archaeology of slavery in the first millennium AD », in J. DAVIS et M. MCCORMICK (dir.) The long morning of medieval Europe: New directions in early medieval studies, Aldershot/Burlington, Ashgate Pub. Co., 2008, p. 33-53 ; Id., «Early European towns: The way of the economy in the Frankish area between dynamism and deceleration, 500-1000 AD », in J. HenNING (dir.) PostRoman towns, trade and settlement in Europe and Byzantium, t. 1, The heirs of the Roman West, Berlin, W. de Gruyter, 2007, p. 3-40 ; Frans Theuws, « Where is the eighth century in the towns of the Meuse valley? », ibid., p. 154-164.

13 - J. HenNING, « Strong rulers - weak economy?... », art. cit., et $I d$. « Early European towns... », art. cit.
} 
Ce point de vue, dont la force est de reposer sur des travaux archéologiques et donc sur l'apport d'une documentation nouvelle, est difficile à rendre compatible avec les tendances de l'historiographie en matière économique depuis les années 1980. Elle pose également des questions d'ajustement avec l'archéologie agraire telle qu'elle se développe en France depuis les années $1980^{14}$.

La périodisation sous-jacente est connue : les $\mathrm{VI}^{\mathrm{e}}-\mathrm{XI}^{\mathrm{e}}$ siècles seraient un moment de contraction démographique et de stagnation économique, aussi bien en ce qui concerne la production que les échanges. Le décollage s'opérerait au XI ${ }^{\mathrm{e}}$ siècle, à la faveur des grands défrichements et de la diffusion d'innovations techniques susceptibles d'avoir bouleversé la vie économique. Elle a été acceptée des années 1930 aux années 1960 et confortée par le jugement très négatif porté sur l'économie carolingienne par l'historiographie des années 1950 et 1960, notamment par Georges Duby et Robert Fossier qui ont professé que l'économie rurale des $\mathrm{VI}^{\mathrm{e}}-\mathrm{X}^{\mathrm{e}}$ siècles était une économie de misère, aux rendements invraisemblablement bas, sans moyens techniques pour l'emporter dans une lutte inégale contre les conditions naturelles ${ }^{15}$. Cette thèse présuppose l'incompétence des élites sociales en matière économique et privilégie l'idée d'un comportement ostentatoire développé à tel point qu'il rendrait impossible tout investissement productif, la richesse disponible étant immédiatement consommée ou redistribuée.

14 - Voir, pour une première approche déjà ancienne : Jean CUISENIER et Rémy GUADAGNIN (dir.), Un village au temps de Charlemagne. Moines et paysans de l'abbaye de Saint-Denis du VII siècle à l'an mil, Paris, Éd. de la Réunion des musées nationaux, 1988. Élisabeth ZADORA-RIO, « Le village des historiens et le village des archéologues », in É. MORNET (dir.), Campagnes médiévales. L'homme et son espace. Études offertes à Robert Fossier, Paris, Publications de la Sorbonne, 1995, p. 145-153. L'étude de la culture matérielle a continuellement progressé : Édith PEYTREMANN, Archéologie de l'habitat rural dans le nord de la France du IV au XII siècle, Saint-Germain-enLaye, AFAM, 2003 ; Élise FAURE-BOUCHARLAT (dir.), Vivre à la campagne au Moyen Âge. L'habitat rural du $V^{e}$ au XII siècle (Bresse, Lyonnais, Dauphiné) d'après les données archéologiques, Lyon, Association lyonnaise pour la promotion de l'archéologie en Rhône-Alpes, 2001 ; Isabelle CATTEDDU, « Le renouvellement des connaissances sur l'habitat et l'espace rural au haut Moyen Âge », in J.-P. Demoule (dir.), L'archéologie préventive dans le monde. Apports de l'archéologie préventive à la connaissance du passé, Paris, La Découverte, 2007, p. 82-101.

15 - Georges DuBY, L'économie rurale et la vie des campagnes dans l'Occident médiéval, France, Angleterre, Empire, $I X^{e}-X V^{e}$ siècles. Essai de synthèse et perspectives de recherches, Paris, Éditions Montaigne, 1962 ; Robert FosSIER, «Les tendances de l'économie: stagnation ou croissance ?», in Nascita dell'Europa ed Europa carolingia : un'equazione da verificare, Spolète, Centro italiano di studi sull' alto medioevo, 1981, p. 261-274. 
Cependant, prenant à contre-pied le point de vue pirennien, Georges Despy ${ }^{16}$, Adriaan Verhulst ${ }^{17}$, Pierre Toubert ${ }^{18}$ et Pierre Bonnassie ${ }^{19}$ ont montré que le capital foncier pouvait engendrer du profit et que le comportement économique des agents devait être considéré comme obéissant à une rationalité minimale. Les gestionnaires cherchent ainsi à tirer le meilleur parti possible de leur allocation de ressources et agissent de manière à assurer en permanence la couverture des besoins alimentaires fondamentaux. Ce ne sont pas de purs consommateurs parasites mais des hommes ayant le souci de maintenir et développer leur richesse ${ }^{20}$. L'attention s'est alors portée sur l'existence et le nombre des marchés ruraux dont la présence sert à évacuer les surplus agricoles lorsque, et, s'il s'en trouve. Ainsi, sur la Meuse, un véritable réseau s'est structuré avant même l'émergence des villes du Nord en tant que puissances productrices et exportatrices de draps. Renversant la problématique héritée d'Henri Pirenne, Georges Despy et Adraan Verhulst montrent qu'il y a, avant l'essor des villes drapantes, une phase d'accumulation reposant sur des échanges locaux animés par la vente des surplus (supervacua) agricoles provenant de domaines exploités de manière beaucoup moins maladroite ou inexperte que ce que l'on avait dit et enseigné.

De son côté, P. Toubert montre, pour l'Italie, l'importance des marchés domaniaux durant l'époque carolingienne et insiste sur l'adaptation de l'instrument monétaire aux besoins de la vie d'échanges, le pouvoir libératoire du denier correspondant aux dépenses susceptibles d'être faites sur les marchés locaux ${ }^{21}$. J.-P. Devroey, enfin, expose l'importance des circulations à l'intérieur des systèmes que forment les grands patrimoines fonciers

16 - Georges DESPY, «Villes et campagnes aux IX et $\mathrm{X}^{\mathrm{e}}$ siècles : l'exemple des pays mosans », Revue du Nord, 50, 1968, p. 145-168.

17 - AdriaanVERHULST, « La genèse du régime domanial classique en France au haut Moyen Âge », in Agricoltura e mondo rurale in Occidente nell'alto medioevo, Spolète, Centro italiano di studi sull' alto medioevo, 1966, p. 135-160.

18 - Pierre Toubert, L'Europe dans sa première croissance. De Charlemagne à l'an mil, Paris, Fayard, 2004 : p. 27-72, p. 73-116.

19 - Pierre BonNASsIE, « La croissance agricole du Haut Moyen Âge dans l'Espagne du Nord-Est et la Gaule du Midi : chronologie, modalités, limites », in CENTRE CUltUREL DE L'ABBAYE DE FlarAn, La croissance économique du haut Moyen Âge, Auch, Comité départemental du tourisme du Gers, 1990, p. 13-35.

20 - L. FELLER, « Introduction... », art. cit., p. 5-30.

21 - Pierre TOUBERT, « Il sistema curtense : la produzione e lo scambio interno in Italia nei secoli VIII, IX e X », in R. ROMANO et U. TUCCI (dir.), Economia naturale, economia monetaria, Turin, G. Einaudi, 1983, p. 5-63. 
monastiques et les relie à l'existence de marchés de consommation, qu'il s'agisse des villes, dont la population est déjà demandeuse en produits alimentaires élaborés, ou de la zone de consommation liée aux trafics de la mer du Nord ${ }^{22}$.

Ces recherches ont abouti, dès la fin des années 1980, à une révision très argumentée de la chronologie : si les $\mathrm{VI}^{\mathrm{e}}$ et $\mathrm{VII}^{\mathrm{e}}$ siècles voient s'opérer un certain nombre de régressions et de reculs, tant au point de vue démographique qu'au point de vue de l'organisation de la production, en revanche, l'apparition du grand domaine durant le VII ${ }^{\mathrm{e}}$ siècle est le signe d'une reprise en mains de la vie économique par les élites politiques et sociales. Elle prélude à une phase de développement, lente et fragile mais réelle, que l'on observe dès le VIII ${ }^{\mathrm{e}}$ siècle. Les structures foncières, les techniques agraires et l'organisation des échanges autorisent à parler d'une croissance du haut Moyen Âge, commencée dès l'époque carolingienne, le XI ${ }^{\mathrm{e}}$ siècle étant un moment d'intensification et d'accélération d'un mouvement commencé deux siècles auparavant ${ }^{23}$. Cette chronologie et ces points de vue concernant la vie économique en général sont difficilement compatibles avec les analyses des résultats obtenus actuellement par l'archéologie des villes du haut Moyen Âge ${ }^{24}$. En l'état actuel des choses, cependant, c'est ainsi que l'on rend compte du plus grand nombre de situations et que l'on explique le plus grand nombre de faits constatés.

Plus récemment, C. Wickham a suggéré d'analyser différemment les questions liées aux échanges entre $\mathrm{VI}^{\mathrm{e}}$ et $\mathrm{IX}^{\mathrm{e}}$ siècle en synthétisant les études portant sur les fossiles directeurs que l'archéologie fournit, en l'espèce, les céramiques. Liant la restriction de l'espace géographique à l'intérieur duquel s'effectuent les échanges commerciaux - indéniable selon lui dès le $\mathrm{V}^{\mathrm{e}}$ siècle - et les nécessités locales, il montre l'existence de réseaux commerciaux régionaux et locaux portant sur des biens pouvant aller du produit de très grand luxe à l'objet de mauvaise qualité mais indispensable à la vie quotidienne ${ }^{25}$. Les céramiques circulent à

22 - Jean-Pierre Devroey, «Les services de transport de l'abbaye de Prüm au IX ${ }^{\mathrm{e}}$ siècle », Revue $d u$ Nord, 61, 1979, p. 543-569 ; Id., «Un monastère dans l'économie d'échanges : les services de transport à l'abbaye de Saint-Germain-des-Prés au IX ${ }^{\mathrm{e}}$ siècle », Annales ESC, 39-3, 1984, p. 570589.

23 - Centre culturel de L'ABBAye de Flaran, La croissance agricole du haut Moyen-Âge. Chronologie, modalités, géographie, Auch, Comité départemental du tourisme du Gers, 1990.

24 - Voir note 13.

25 - C. WiCKHAM, Framing the early Middle Ages..., op. cit., p. 819-824. Voir aussi les travaux de Ghislaine NOYE, «Economia e società nella Calabria bizantina », in A. PlaCANICA (dir.), Storia della Calabria medievale, t. I, Quadri generali, Rome, Gangemi, 2001, p. 579-656. 
l'intérieur de zones restreintes mais très actives. Elles peuvent parfois être des productions de très haute qualité et de grand prix. En revanche, les grands circuits animés par une production industrielle de masse, ceux de la céramique sigillée, ont disparu dès le VII siècle. Production et commerce locaux ont permis de pallier l'insuffisance, voire l'effondrement, de ces réseaux dont la survie est effectivement très longue ${ }^{26}$.

Enfin, C. Wickham estime et démontre que l'apparition du grand domaine carolingien est synonyme d'intensification du travail et, donc, d'augmentation tendancielle de la production agricole. Le grand domaine est une structure coercitive dont l'efficacité est réelle dans l'encadrement des travailleurs. Sa construction a impliqué une mobilisation des élites sociales et leur implication dans la vie économique dans des proportions telles qu'il est difficile d'imaginer qu'elles n'y aient pas vu un intérêt immédiat. L'organisation de la réserve seigneuriale est telle que, même si la productivité du travail est basse et le rendement des terres faibles, il faut supposer que du surplus est produit et que celui-ci trouve un exutoire sur des marchés. La logique de la documentation et celle des argumentations les plus sophistiquées poussent désormais à accepter l'existence d'échanges locaux relativement intenses durant la période allant ${\mathrm{du} \mathrm{VI}^{\mathrm{e}}}^{\mathrm{au}} \mathrm{X}^{\mathrm{e}}$ siècle. En conséquence, il faut également admettre celle de lieux physiques, les marchés, où l'on compare les marchandises avant de les échanger dans le cadre d'un véritable commerce susceptible de contribuer de façon non marginale aux revenus des acteurs.

Il faut maintenant de poser la question de leur fonctionnement en examinant ce que nous savons des procédures de formation des prix, qu'il s'agisse de ceux de la terre ou de ceux des biens meubles.

\section{Marchés fonciers et marchés du haut Moyen Âge}

Entre $\mathrm{VI}^{\mathrm{e}}$ et $\mathrm{XII}^{\mathrm{e}}$ siècles, l'organisation des échanges entre les hommes ne saurait être considérée comme régie par les marchés au sens où Adam Smith l'entendait: cette affirmation, qui est devenue de l'ordre du truisme, doit être rappelée afin de pouvoir la mettre à l'épreuve de la réflexion et des faits et de voir si elle peut s'accommoder d'actions

${ }^{26}$ - Voir sur la question de cette articulation les travaux de S. GeLICHI sur la plaine du Pô : Sauro GelichI, «La richezza nella società longobarda », in J.-P. DeVroey, L. Feller et R. Le JAN (dir.) Les élites et la richesse..., op. cit., p. 159-183; Gian Pietro BROGIOLO et Alexandra CHAVARRIA ARnAu, Aristocrazie e campagne nell'Occidente da Costantino a Carlo Magno, Florence, All'insegna del giglio, 2005. 
rationnelles et, si tel est le cas, comment les deux ordres peuvent être rendus compatibles l'un avec l'autre. L'imbrication ou l'encastrement de l'économique dans le social aurait pour conséquence ultime que les agents ne chercheraient pas nécessairement à maximiser leur profit mais poursuivraient, en procédant à des échanges de choses contre d'autres choses ou contre de l'argent, des buts extrêmement nombreux où la réalisation d'un gain ne serait ni nécessaire ni même parfois souhaitable. L'objet ayant suscité le plus de réflexion à ce propos est sans conteste possible la terre et les transactions dont elle fait l'objet, parce qu'elle fournit, en l'absence de listes de prix portant soit sur des produits manufacturés soit sur les céréales, des indications de surface, de localisation et de valeur et permet de construire des séries. Les caractères propres de cet objet paradoxal qu'est la terre lorsqu'on la considère comme une marchandise sont connus depuis longtemps et ont fait l'objet de nombreuses analyses ${ }^{27}$ : faire de la terre une marchandise, alors même qu'elle est aussi un instrument de production et qu'elle est de surcroît le support matériel du capital symbolique de son possesseur, pose des problèmes théoriques et pratiques dont l'analyse est devenue classique ${ }^{28}$. D'un autre côté, la terre s'achète et se vend et la description des conditions dans lesquelles s'opèrent les transactions comme les négociations entre individus et groupes auxquelles il donne lieu permet d'enrichir notre perception de la vie d'échanges.

Giovanni Levi, Gérard Delille et Chris Wickham ont exploré ces voies pour les époques moderne et médiévale ${ }^{29}$ et, depuis les années 1980, le marché de la terre a été utilisé comme moyen d'approche de l'organisation générale des échanges. Examiner les procédures par lesquelles les terres s'échangent permet en effet de formuler la question de la formation des prix d'un bien exceptionnel mais pour lequel on dispose de données chiffrées susceptibles de

27 - K. POlAnYI, La grande transformation..., op. cit., p. 238 sq. ; Chris WiCKHAM, «Vendite di terra e mercato della terra in Toscana nel secolo XI », Quaderni Storici, 65, 1987, p. 355-377 ; Id., The mountains and the city: The Tuscan Appennines in the early Middle Ages, Oxford/New York, Clarendon Press/Oxford University Press, 1988 ; L. FELLER, «Introduction... », art. cit. ; Florence WEBER, « De l'anthropologie économique à l'ethnographie des transactions », in J.-P. DEVROEY, L. FELLER et R. LE JAN (dir.) Les élites et la richesse..., op. cit., p. 29-48.

28 - Simonetta CAVAciocchi (dir.), Il mercato della terra. Secc. 13-18, Grassina, Le Monnier, 2004.

29 - Giovanni LEVI, Le pouvoir au village. Histoire d'un exorciste dans le Piémont du XVII siècle, Paris, Gallimard, [1985] 1989 ; Gérard DeliLle, « Dot des filles et circulation des biens dans les Pouilles aux XVII ${ }^{\mathrm{e}}$ et XVIII ${ }^{\mathrm{e}}$ siècles », Mélanges de l'École Française de Rome, Moyen Âge, 95-1, 1983, p. 195-224; Id., Famille et propriété dans le royaume de Naples (XVe-XIX siècles), Rome/Paris, École française de Rome/Éd. de l'EHESS, 1985. 
constituer des séries. Ainsi, pour les Abruzzes de la fin du haut Moyen Âge, il a été possible, à partir de l'étude des prix du foncier, de proposer une périodisation de la vie économique et d'étudier les différents mécanismes et intérêts ou les différentes forces à l'œuvre dans une société rurale entre $\mathrm{IX}^{\mathrm{e}}$ et $\mathrm{XII}^{\mathrm{e}}$ siècle ${ }^{30}$. Parler de prix suppose normalement que l'on admette l'existence et le fonctionnement de mécanismes impersonnels incluant l'offre, la demande et leur rencontre. Or, il existe aussi, de manière indubitable, une genèse sociale des prix parce que les relations personnelles pèsent d'un poids considérable dans la définition même de l'échange monétaire.

Or, si les études des années 1970-1980 ont toujours insisté, à raison, sur le fait que les échanges médiévaux se caractérisaient par le nombre et la complexité des interventions à caractère non économique sur les transactions, il n'en demeure pas moins que les séries chiffrées que l'on peut constituer à partir des indications de surface et de prix tout d'abord, quelque rudimentaires qu'elles semblent, sont tout à fait utilisables. Elles permettent d'établir des évolutions à la hausse ou à la baisse sur le long terme. Grâce à elles il est aussi possible, à condition de recourir à un appareil statistique, de comprendre comment se forment les prix en fonction des catégories de biens considérés. C'est ce qui a été entrepris, voici quelques années, au sein d'un programme sur le fonctionnement du marché de la terre, avec l'étude d'un dossier sur le patrimoine d'un alleutier italien, vivant dans les Abruzzes au IX ${ }^{\mathrm{e}}$ siècle ${ }^{31}$. On me permettra d'en présenter brièvement les acquis en en généralisant la portée.

L'analyse économétrique à laquelle on s'est livré dans La fortune de Karol a permis d'isoler les variables qui interviennent dans le processus et de proposer une équation rendant compte de la variation des prix en fonction des éléments donnés par la documentation : la présence de terres arables, de vignes, de parcelles spécialisées, de maisons, d'enclos détermine des valeurs différentes et, surtout, des modes d'estimation différents selon les biens

\footnotetext{
30 - Laurent Feller, Les Abruzzes médiévales. Territoire, économie et société en Italie centrale du $I X^{e}$ au XII siècle, Rome, École française de Rome, 1998, p. 386-415.

31 - Laurent FELler, Agnès Gramain et Florence WeBEr, La fortune de Karol. Marché de la terre et liens personnels dans les Abruzzes au haut Moyen Âge, Rome, École française de Rome, 2005. Cette recherche s'insérait dans le programme «Le marché de la terre au Moyen Âge » promu entre 1998 et 2001 par l'UMR 8589/LAMOP et dont les résultats ont été publiés en 2005 sous le même titre. Le volume, qui aurait dû être inséré dans la publication a été édité à part du fait de l'importance qu'il avait prise.
} 
échangés ${ }^{32}$. Toutes ces indications font sens et il est possible de mobiliser les indications chiffrées, dans ce cas d'espèce celles données par un cartulaire, pour mesurer des tendances et des variations. Le comportement des hommes du haut Moyen Âge peut aussi être décrit grâce à des analyses économétriques qui, à condition de n'être pas exclusives dans leur approche, contribuent à éclairer les attitudes face aux échanges et à leur organisation concrète. En l'occurrence, il a ainsi été montré que les transactions foncières donnaient lieu à de véritables marchés, segmentés cependant en fonction de la nature des biens en jeu.

Ce n'est cependant pas le seul angle d'attaque légitime ou nécessaire. Le dossier de Karol comportait en effet des renseignements complets et précis sur les alliances matrimoniales, les situations hiérarchiques et l'évolution des patrimoines : il était possible de mettre les uns en rapport avec les autres. Si l'approche économétrique a permis dans ce cas d'établir les règles de fonctionnement de marchés et de décrire leur évolution dans le temps, ce qui est un résultat positif évidemment appréciable, elle ne rendait cependant pas compte de l'autre aspect des transactions, c'est-à-dire de leur insertion dans un réseau d'actions et de relations interpersonnelles : l'examen des prix ne saurait se substituer à une analyse portant sur les liens existant entre les hommes avant et après la transaction. Les acteurs ne cessent pas en effet d'être en relation après avoir effectué l'ensemble des gestes qui concluent la vente. Appartenant à des groupes de parenté, à des voisinages, à des réseaux hiérarchiques, ils continuent après la transaction d'entretenir des relations, sauf dans le cas, tout de même rare, où la vente précède un départ et est effectuée à la fois pour solder les comptes antérieurs et apurer les dettes ou pour obtenir, au moment de partir, des liquidités permettant d'envisager une réinstallation ailleurs. La continuité des relations entre les parties transactantes n'empêche cependant pas une suspension des autres enjeux que celui du prix au moment même où l'échange s'effectue ${ }^{33}$.

L'approche, alors, devient ethnographique, afin de déterminer et de qualifier ce que font effectivement les auteurs en faisant circuler la propriété du sol. Il est nécessaire d'intégrer à l'analyse des éléments qui ne sont pas mesurables et de s'intéresser aussi bien aux relations de parenté qu'à celles de voisinage ou encore à la position respective des actants dans la hiérarchie politique de la communauté ${ }^{34}$.

\footnotetext{
32 - L. Feller, A. Gramain et F. Weber, La fortune de Karol..., op. cit., p. 35-51. Ce qui suit, d'après Laurent FELLER, « Histoire du Moyen Âge et histoire économique... », art. cit., p. 39-60.

33 - F. WEBER, « De l'anthropologie économique à l'ethnographie des transactions... », art. cit.

34 - G. DeliLle, « Dot des filles... », art. cit. ; G. LeVI, Le pouvoir au village..., op. cit.
} 
La recherche d'une alliance de mariage, d'une proximité politique ou la construction d'une relation amicale ont ainsi une relation avec le niveau des prix ou la nature de la contrepartie demandée en échange de la terre, puisque ce n'est pas tant l'utilité d'un bien qui est en jeu que l'institution ou la consolidation d'un lien. La relation de marché est donc inversée, l'objet n'étant que le vecteur du dialogue établi entre les parties afin de construire leur relation : il n'est pas désiré pour lui-même mais pour ce qu'il peut représenter. C'est donc l'acte d'échange qui importe, non la valeur circulant. C. Wickham a ainsi montré que, dans la Toscane du XI ${ }^{\mathrm{e}}$ siècle, la circulation des parcelles de terre, qui était intense, était provoquée non par le désir d'accroître son revenu en augmentant les capacités productives de l'exploitation, mais par celui d'établir et d'entretenir des relations avec le plus grand nombre possible de membres de la communauté villageoise ${ }^{35}$.

Dans ces conditions, l'attention doit alors se porter à la fois sur la description de la transaction et sur les moyens utilisés pour la solder : une analyse très précise des formulaires et de leurs variations donne des résultats non négligeables, du moins dans le contexte italien des $\mathrm{IX}^{\mathrm{e}}-\mathrm{XI}^{\mathrm{e}}$ siècles ${ }^{36}$. L'absence ou la présence de certains renseignements dans les actes de mutation éclaire en effet la finalité des transactions : les notaires, par exemple, ne décrivent pas la même chose suivant qu'il s'agit d'exploitations ou de simples parcelles ${ }^{37}$. Alors qu'ils donnent toujours les confronts des parcelles, ils sont beaucoup plus vagues sur les limites des exploitations lorsqu'elles sont vendues d'un seul bloc. Les moyens de paiement utilisés, de leur côté, permettent aussi de dire quelque chose de la nature de l'opération en cours, l'emploi de numéraire et celui d'objets ou de bétail ne signifiant pas la même chose. Le paiement effectué à l'aide d'un objet matériel n'a pas la neutralité apparente du versement d'une somme d'argent et dénonce aussitôt la relation dont l'objet choisi pour le transfert établit la nature. Dans la société agraire du haut Moyen Âge, seul un guerrier peut avoir besoin d'un cheval ou d'une épée et l'exiger en paiement d'un bien foncier payé. Seul un exploitant agricole direct, ayant besoin de la force de traction d'un animal, a intérêt à obtenir un bœuf en contrepartie d'une parcelle. Ces affirmations sont au demeurant réversibles, tout comme l'acte d'achat, dont la symétrie doit également être considérée : la vente d'une terre correspond dans ces cas à l'achat d'un cheval, d'une épée ou d'un bœuf, ce qui investit la terre d'une fonction

\footnotetext{
35 - C. WICKHAM, «Vendite di terra... », art. cit.

36 - E. HuERTAS, « La rente foncière à Pistoia $\left(11^{\mathrm{e}-} 12^{\mathrm{e}}\right.$ siècle $)$. Pratiques notariales et histoire économique », thèse, sous la direction de L. Feller, université Paris-Est, 2008.

37 - L. Feller, A. Gramain et F. Weber, La fortune de Karol..., op. cit., p. 85-91.
} 
qu'elle n'a pas de façon évidente, celle de réserve de valeur, à côté de sa fonction d'instrument de production et de support matériel du statut, du prestige et du rang de l'individu ${ }^{38}$.

La présence ou l'absence d'une mesure de la valeur de l'objet échangé, en dehors même de l'indication du medium employé pour solder la transaction, est un autre paramètre permettant là encore de qualifier la relation instaurée autour de l'interaction décrite par l'acte de vente. Dans ces cas, la mention d'un prix ne signifie pas qu'un objet monétaire a changé de main, ni même d'ailleurs que la transaction a donné lieu à une évaluation, mais simplement qu'une contrepartie a été offerte au cours de l'échange. On est bien là dans le cadre d'échanges non commerciaux qui ne disposent cependant pas d'un autre lexique que celui de l'échange marchand pour décrire les opérations. Le système sémantique de l'échange est indéterminé et l'échange marchand se distingue mal de l'échange rituel.

Ainsi, une terre dont la superficie est ou n'est pas donnée peut être échangée contre un bien meuble qui, lui-même, peut être ou n'être pas évalué ni même parfois précisément décrit, les formules, très souples, permettant une infinité de possibilités, depuis celle, précise, donnant une valeur à la chose échangée (c'est pourquoi je te donne une épée, un cheval, une pièce de toile, un bouc, un soc de charrue, valant tant de sous). Elle peut aussi bien n'être pas donnée, ou bien être aléatoire : je te donne du bétail et des grains qui te permettent de te tirer d'une période de famine ou encore, je te donne des biens m'appartenant pour telle contrevaleur (de mea mobilia valente tot). Une série de combinatoires peut ainsi être établie qui permet de donner un sens à la transaction effectuée, non pas en s'intéressant à son prix seul, mais en analysant l'ensemble des éléments la constituant, les objets mobilisés devant obligatoirement être considérés comme faisant sens.

Les analyses ainsi faites permettent de différencier plusieurs marchés aux règles distinctes, le marché des parcelles et celui des exploitations étant les principaux, et plusieurs finalités : échange marchand, transaction dont le but est l'évaluation du bien avant un partage, transactions montrant l'établissement ou la consolidation d'un lien de clientèle, achats

\footnotetext{
38 - Timothy REUTER, « Property transactions and social relations between rulers, bishops and nobles in early eleventh-century Saxony: The evidence of the Vita Meinwerci », in W. DAVIES et P. FOURACRE (dir.), Property and power in the early Middle Ages, Cambridge, Cambridge University Press, 1995, p. 165-199.
} 
préparant un mariage ${ }^{39}$. Ainsi, il devient licite d'utiliser la contrepartie offerte dans un échange comme un indicateur du type de relations existant entre parties ${ }^{40}$.

En revanche, une transaction soldée en numéraire, portant sur un bien précisément décrit et mesuré, dont les confronts et la superficie sont précisément donnés, est un bien estimé auquel une valeur est donc attribuée. Celle-ci ne peut pas être considérée comme arbitraire : elle décrit une capacité productive, la valeur des bâtiments d'exploitation, la proximité des voies de communication. La terre est alors considérée principalement comme un moyen de production et non comme le vecteur du pouvoir ou du prestige. Inversement, dès lors, les transactions soldées avec des objets, dans lesquelles le bien offert n'est pas décrit et où les objets cédés en échange ne sont ni décrits ni évalués, ont toute chance d'aboutir à la constitution d'une relation de clientèle ou de dépendance. Vendre une terre en période de famine, lorsque celle-ci est le seul titre qui demeure à la disposition des paysans afin d'accéder au marché de la nourriture, revient en effet à se placer sous la responsabilité de l'acheteur: si la vente est compensée par de la nourriture, elle établit le lien fondamental et primitif unissant le seigneur, celui qui donne du pain, le hlaford saxon, à celui qui désormais dépend de sa générosité pour sa survie. Le droit d'exploiter la terre dont la propriété est transmise peut bien demeurer entre les mains du vendeur par le biais de la tenure : l'acte de vente aura été en même temps un acte d'entrée en dépendance, la reconnaissance de la domination d'un supérieur hiérarchique.

On peut ainsi, en examinant les procédures d'évaluation ou de mesure ainsi que les moyens de paiement, rendre compte d'un grand nombre de situations en introduisant de la complexité et en évitant de réduire les questions posées par les échanges fonciers à un simple «cela n'est rien d'autre que», par exemple rien d'autre qu'une forme particulière de rationalisation de choix individuel effectué sous contrainte ${ }^{41}$.

Il est possible enfin de distinguer, dans des séries de transactions, celles qui ont à voir avec l'échange marchand et les mettre à part. Dans un très grand nombre de cas, en effet, des valeurs sont en cause et l'on ne peut renvoyer les échanges à une relation interpersonnelle particulière. Ce sont bel et bien des questions d'estimation et de mesure de la valeur qui sont

\footnotetext{
39 - F. WEBER, « De l'anthropologie économique à l'ethnographie des transactions... », art. cit.

40 - L. Feller, A. Gramain et F. WeBer, La fortune de Karol..., op. cit., p. 85-92.

41 - Voir, sur les inconvénients du «nothing but» et de son symétrique, la théorie des «mondes hostiles » : Viviana ZELIZER, «Transactions intimes », Genèses. Sciences sociales et Histoire, 42, 2001, p. 121-144.
} 
traitées et que les contemporains ont, en leur temps, affrontées. Si les choses ont souvent un rapport étroit avec le lien social, elles ont aussi à voir avec la valeur et sa mesure.

Ces résultats peuvent être mobilisés pour étudier les échanges portant sur des biens meubles et tenter d'apercevoir les règles qui président à la formation des prix : il est légitime d'étendre les réflexions faites à partir de l'étude d'un objet complexe et protéiforme à d'autres objets, en apparence plus simples, en l'occurrence les produits alimentaires et artisanaux. Cela revient à atténuer la spécificité des échanges fonciers et à admettre que des règles analogues président à tous les types de transactions.

\section{Échanger des biens meubles}

Le cadre ne change pas fondamentalement, les relations interpersonnelles continuant ici de jouer un rôle essentiel : voisinage, amitié, parenté ne sont pas, bien au contraire, mis entre parenthèses ou hors-jeu, suspendus, lors de tous les cas, même s'ils le sont parfois. Il faut cependant préciser les choses, parce que nous avons affaire à des institutions qui ont pour nom marché, monnaies, poids et mesures, justice, et que ce sont elles qu'il faut s'efforcer d'apercevoir à travers les transactions portant sur des objets.

Les produits changent de main sur des lieux précis, les marchés, où vendeurs et acheteurs, offreurs et demandeurs se rencontrent physiquement. Ces lieux sont mal connus. On sait qu'ils existent et qu'ils font l'objet d'un soin particulier et soutenu de la part des souverains. On ne sait pas trop comment ils fonctionnent concrètement, comment la police en est assurée, comment les prix s'y forment et selon quels critères. L'impression générale laissée par la documentation dès l'époque carolingienne est cependant celle d'une familiarité des hommes avec les lieux de l'échange. Il reste à savoir selon quelles règles celui-ci s'effectuait, quels outils étaient utilisés et quels effets économiques ils avaient. Matthieu Arnoux a fait une enquête rapide sur les mots nundinae et mercatum sur le site de l'ARTEM (Atelier de Recherches sur les Textes Médiévaux) et a pu voir que sur 5000 chartes conservées en original dans les archives françaises et datant d'avant 1121, 160 environ mentionnaient le marché mais toujours dans un contexte juridique, de transmission de droits et dans un contexte fiscal de prélèvements de tonlieux ${ }^{42}$. Les renseignements précis et concrets sont difficiles à obtenir. Ils sont rares aussi.

\footnotetext{
42 - M. ARNOUX, « Vérité et question des marchés médiévaux », art. cit. Les documents en question sont désormais en ligne : http://www.cn-telma.fr/originaux/index.
} 
Toutefois, les rencontres que sont les marchés doivent obéir à des règles dont les principes soient acceptés par tous à un moment donné et dans un lieu donné. Il est tout d'abord nécessaire que l'on s'accorde par convention sur les rites qui organisent l'échange : exposition de la marchandise, inspection par les clients, négociation sur le prix, signes de l'accord (toper, par exemple), versement du prix convenu, cession de l'objet désiré qui est finalement emporté. Il y a là tout un langage à instituer et à maîtriser et la série des actions à effectuer, qui nous est familière, n'a évidemment rien de naturel. Elle marque un accord sur la façon dont on résout la question des deux désirs concurrents, celui de l'acquéreur d'obtenir un objet, celui du vendeur d'obtenir en échange quelque chose d'une valeur égale ou supérieure. Si l'on ne connaît pas précisément les rites accompagnant la vente d'objets ou de biens meubles, en revanche ceux accompagnant les transferts de terres sont fort bien connus et ont déjà fait l'objet d'analyses, dont celle, particulièrement profonde, de Jacques Le Goff ${ }^{43}$ : des objets doivent passer de main en main pour signifier le changement de propriété. Il peut s'agir de gants, de branches d'arbres, de fétus de paille, voire de mottes de terre. Ils rendent publics le transfert de propriété, voire le changement de destination de la terre cédée qui, lorsqu'elle passe d'un laïc à un établissement ecclésiastique, cesse d'être exclusivement un objet de production pour être incluse parmi les biens sacrés, que l'on ne peut plus vendre et qui échappent donc désormais aux règles de l'évaluation. Nous verrons que, pourtant, les clercs savent évaluer et mesurer précisément la valeur d'échange même des terres inaliénables qu'ils détiennent et que, même, en fonction des nécessités, ils n'hésitent pas à vendre ou échanger les biens que leurs établissements possèdent.

De ces rites qui se concluent par le paiement et la prise de possession de l'objet, nous ne savons pratiquement rien, voire absolument rien, dès lors qu'il s'agit de biens meubles. Ce que nous savons se limite pratiquement à cette injonction que l'on trouve dans le concile de Paris de 829 : Si vis emere, fer pretium et tolle, «Si tu veux acheter, apporte le prix et emporte la marchandise ${ }^{44} »$, ce qui est, dans le contexte des actes du concile, une façon de refuser de faire crédit à l'acheteur en exigeant de lui qu'il paie comptant mais ne dit pas grand-chose sur la manière dont il faut ferre pretium et ne dit rien non plus sur ce qu'il faut entendre par

\footnotetext{
43 - Jacques Le Goff, «Le rituel symbolique de la vassalité », Pour un autre Moyen Âge. Temps, travail et culture en Occident: 18 essais, Paris, Gallimard, 1977, p. 349-420.

44 - Monumenta Germaniae Historica, Concilia aevi Karolini [742-842], éd. par A. Werminghoff, Berlin, Weidmann, 1997, liv. I, chap. 53, p. 645.
} 
pretium: de l'argent ou des contreparties matérielles ? Des monnaies ou des objets dont la valeur équilibre aux yeux des parties celle de l'objet cédé ?

Seules les indications que nous pouvons avoir sur les moyens et les méthodes de paiement sont pour nous utilisables : par exemple, le fait de recourir à des baguettes de taille dans le cadre d'un échange effectué sur le marché ou, plus souvent, dans une échoppe, implique que l'on est en train de recourir à une forme de crédit, et indique que les parties se connaissent et se fréquentent, au moins dans le cadre de l'échange, c'est-à-dire qu'acheteurs et vendeurs, dans ce cas créditeurs et débiteurs, ne sont pas l'un pour l'autre des inconnus ${ }^{45}$. Cela influe naturellement sur la question des prix qui ne sont plus seulement la résultante de l'offre et de la demande, mais pour la compréhension desquels il faut réinsérer les relations interpersonnelles existantes ou établies à travers la succession des échanges et ce que l'on peut appeler la «relation de crédit». La relation de marché doit alors être considérée non comme une rencontre occasionnelle de deux acteurs étrangers l'un à l'autre et qui ne sont en contact que le temps de la transaction, mais comme l'une des modalités des relations existant à l'intérieur d'un groupe humain restreint. À travers elle, certaines formes de la domination sociale et économique s'expriment, à côté d'autres disant quelque chose de la solidarité entre les individus ou les groupes : le crédit consenti, s'il ne porte pas intérêt, ou s'il porte un intérêt très bas, peut relever de la solidarité à travers l'exercice d'une charité active. Les législations carolingiennes portant sur le crédit ne disent pas autre chose ${ }^{46}$. Il peut également, d'ailleurs, relever des banales conventions sur les délais de paiement qui entourent normalement les transactions.

Le second accord devant intervenir porte sur le recours aux moyens de paiement. Celuici doit faire l'objet d'un consensus de tous les participants, quelle que soit la forme que prend la monnaie : l'essentiel est que tous s'accordent et qu'il y ait un instrument servant de medium de l'échange et d'instrument de mesure de la valeur des objets. Ce peut bien alors être une certaine quantité de grains : il suffit que, par convention, la valeur en soit déterminée

45 - Ludolf Kuchenbuch, «Les baguettes de taille au Moyen Âge : un moyen de calcul sans écriture ? » in N. COQUERY, F. MENANT et F. WEBER (dir.), Écrire, compter, mesurer. Vers une histoire des rationalités pratiques, Paris, Éd. rue d'Ulm, 2006, p. 113-142.

46 - François BougARD, «Le crédit dans l'Occident du Haut Moyen Âge : documentation et pratique », in J.-P. Devroey, L. Feller et R. LE JAN (dir.) Les élites et la richesse..., op. cit., p. $439-478$. 
et acceptée pour régler des échanges ${ }^{47}$. Ce peut aussi être un animal, pourvu qu'il soit évalué (un bœuf valant tant de sous). L'attention portée par les Carolingiens à la monnaie et aux conditions de mise en circulation de celle-ci montre que, dès le VIII ${ }^{\mathrm{e}}$ siècle, les autorités souveraines ont conscience de la nécessité de cet accord. À peine la réforme monétaire lancée, Charlemagne ordonne que la nouvelle monnaie ait cours sur les marchés et qu'en aucun cas on ne puisse la refuser. Il le fait par exemple dans le capitulaire de Mantoue en 781 et réitère l'ordre dans celui de Francfort en $794^{48}$. Cela ne signifie naturellement pas qu'il y ait assez de pièces d'argent en circulation, ni que les agents y aient toujours recours pour solder leurs achats, mais simplement qu'ils existent au moins comme instruments de mesure de la valeur et d'énonciation des prix ${ }^{49}$.

La mention d'un pretium dans les textes ne veut cependant pas nécessairement dire, comme on l'a vu à propos des échanges portant sur des biens fonciers, que la transaction se solde en argent monnayé. Le pretium peut tout aussi bien être, comme il arrive dans nombre d'actes de vente, un objet non monétaire dont la valeur n'est pas toujours donnée et qui sert de contrepartie dans l'échange, plaçant celui-ci dans une sphère différente de celle de l'échange marchand ${ }^{50}$. C'est ce qui se passe en Toscane entre $\mathrm{VI}^{\mathrm{e}}$ et XII ${ }^{\mathrm{e}}$ siècle, où, lorsque des objets changent de possesseurs, des anneaux sont offerts en launegild, en contre-don obligatoire ${ }^{51}$.

47 - Jean GAUTIER-DALChE, «L'histoire monétaire de l'Espagne septentrionale du IX ${ }^{\mathrm{e}}$ siècle au $\mathrm{XII}^{\mathrm{e}}$ siècle. Quelques réflexions sur divers problèmes », Anuario de Estudios Medievales, 6, 1969, p. 43-96; Wendy DAVIES, «Sale, price and valuation in Galicia and Castile-León in the tenth century », Early Medieval Europe, 11-2, 2002, p. 149-174.

48 - Monumenta Germaniae HistoricA, Capitularia regum Francorum, t. I, Capitularia Merowingica, éd. par A. Boretius, Hannovre, Hahn, 1960, n 89, p. 191. Paul FreEdmAN, « Spices and late-Medieval European ideas of scarcity and value », Speculum, 80-4, 2005, p. 1209-1227.

49 - Sur la question des émissions monétaires, voir Simon COUPLAND, Carolingian coinage and the Vikings: Studies on power and trade in the 9th century, Aldershot/Burlington, Ashgate/Variorum, 2007, chap. I et III. Sur les usages concrets et quotidiens des monnaies : Alessia RovelLI, « Coins and trade in early medieval Italy », Early Medieval Europe, 17-1, 2009, p. 45-76.

50 - L. Feller, A. Gramain et F. Weber, La fortune de Karol..., op. cit., p. 78-82 et p. 131-142. Cartulaire de Casauria, BnF, ms. Lat. 5411, fol. 83vº-84 (a. 873) : (...) pretium ab ipso Romano, abbate, caballos duo cum paratura sua in pretium definitum. «J'ai reçu en prix, de toi Romain, abbé, deux chevaux avec leur équipement, en prix convenu ».

51 - Gabriella GARZELLA, «La 'moneta sostitutiva' nei documenti pisani dei secoli XI e XII : un problema risolto ? », in G. GARzella, M. L. CeCCARelli LemUt et B. CAsini (dir.), Studi sugli strumenti di scambio a Pisa nel medioevo, Pise, Pacini, 1979, p. 3-45. Chris Wickham, «Compulsory gift exchange in Lombard Italy (650-1150)», in W. DAVIES et P. FourACRE (dir.), 
Mais ces anneaux peuvent être également de véritables objets monétaires, en ceci que leur poids et leur titre sont connus et peuvent faire l'objet d'une mise en relation avec l'objet échangé. Leur signification ne peut donc pas être exclusivement symbolique : en fonction du négoce en cause, ils servent à autre chose qu'à offrir un contre-don obligatoire dans un échange. Celui-ci peut, au demeurant, être très complexe et comporter des éléments monétaires formant un équivalent de prix à côté de cadeaux considérés comme obligatoires et constituant le launegild.

Les questions que pose la réalité de la circulation monétaire durant le haut Moyen Âge, de son importance et de sa nature même, sont immenses et difficiles : on ne les ouvrira pas ici sauf à souligner qu'à partir du VIII ${ }^{\mathrm{e}}$ siècle, le denier fournit un moyen d'évaluation universel utilisé dans toute l'Europe, même s'il n'est pas nécessairement utilisé pour solder effectivement les échanges. Il conserve sa place après la disparition de l'empire et l'éclatement de tout pouvoir central, ce qui constitue l'un des paradoxes les plus étonnants de la période dite féodale ${ }^{52}$. De plus, son prestige demeure tel qu'il est imité partout. Le système d'évaluation imposé par les Carolingiens, reposant sur la livre, le sou et le denier, où seul le denier et, parfois, son sous-multiple, l'obole, est une monnaie réelle, demeure ainsi étonnamment vivant et solide.

Par-derrière ce truisme se cache une question assez simple à formuler mais dont la réponse est difficile à apporter : comment le développement économique de l'Europe a-t-il pu s'effectuer alors que les moyens de paiement semblent insuffisants en quantité comme en qualité ? Les techniques de substitution, comme le recours à des objets non monétaires auxquels une valeur est artificiellement affectée, doivent connaître assez vite des limites. La maîtrise, finalement assez bonne du crédit et de certains de ses instruments, fournit sans doute une partie de l'explication ${ }^{53}$, mais il y a encore beaucoup de chemin à faire pour comprendre ce qui s'est exactement joué en Europe entre $\mathrm{IX}^{\mathrm{e}}$ et $\mathrm{XII}^{\mathrm{e}}$ siècle, pour être large, autour de la

The languages of gift in the early Middle Ages, Cambridge, Cambridge University Press, 2010, p. 193-216, spécialement p. 198 et 207-210. Sur la distinction entre prix et contre-don, voir W. DAVIES, « Sale, price and valuation... », art. cit., p. 156-157 ; L. FELLER, A. GRAMAIN et F. WEBER, La fortune de Karol..., op. cit., p. 78-85.

52 - P. TOUBERT, « Il sistema curtense... », art. cit. ; Voir Alessia RovELLI, « Some considerations on the coinage of Lombard and Carolingian Italy », in I. HANSEN et C. WICKHAM (dir.), The long eighth century, Leyde, Brill, 2000, p. 195-224 qui exprime un désaccord de fond avec P. Toubert sur le pouvoir libératoire du denier.

53 - F. BougARD, « Le crédit dans l'Occident du Haut Moyen Âge... », art. cit., note 46. 
question monétaire. L'œuvre carolingienne, en tout cas, doit être considérée comme d'autant plus fondamentale en cette matière qu'elle fournit manifestement le cadre mental à l'intérieur duquel producteurs et consommateurs se situaient lorsqu'ils devaient échanger.

La troisième règle de ce jeu est l'usage de poids et de mesures communs aux parties. Celles-ci permettent d'avoir une idée précise des quantités mises en jeu, ce qui est évidemment indispensable à toute forme d'échanges. On sait que les Carolingiens ont apporté une certaine attention à cette question, à côté du soin qu'ils ont eu des monnaies. La polémique qui, dans les années 1970, a opposé Michel Rouche à Jean-Claude Hocquet portait précisément sur ces questions de mesure ${ }^{54}$. Diverses allusions permettent de déduire qu'il y a $\mathrm{eu}$, à la fin du VIII ${ }^{\mathrm{e}}$ siècle ou au début du $\mathrm{IX}^{\mathrm{e}}$ siècle, une réforme des poids et des mesures, sans que cela éclaire de façon définitive notre connaissance de la question. Celle-ci est présente dans l'érudition depuis les Prolégomènes donnés en guise d'introduction à l'édition du polyptyque d'Irminon par Benjamin Guérard, sans que le problème qu'il prétendait résoudre, celui de la contenance d'une mesure volumétrique, le muid, ait été résolu de façon satisfaisante.

Dès 794, le capitulaire de Francfort, par lequel Charlemagne impose un maximum des prix du blé, propose une équivalence muid-denier qui suppose, pour être comprise, que le denier nouvellement frappé soit accepté partout-et que le muid volumétrique soit également une mesure universelle : la norme, autrement, ne ferait pas sens. Un peu plus tard, en 822 , les statuts d'Adalhard de Corbie mentionnent l'existence d'un muid public nouvellement institué : c'est en y recourant que l'abbé calcule les rations de ses moines ${ }^{55}$. Les décisions prises par Charlemagne et son fils en ce qui concerne les mesures ont reçu un commencement

54 - Michel Rouche, «La faim à l'époque carolingienne. Essai sur quelques types de rations alimentaires », Revue Historique, 250, 1973, p. 295-320 ; Jean-Claude HoCQUET, « Le pain, le vin et la juste mesure à la table des moines carolingiens ", Annales ESC, 40-3, 1985, p. 661-686. Bernard GARNIER, Jean-Claude HOCQUET et Denis WORONOFF (dir.), Introduction à la métrologie historique, Paris, Economica, 1989. Jean-Pierre DEVROEY, «Units of measurement in the early medieval economy: The example of Caroligian food ration », French History, 1-1, 1987, p. 68-92.

55 - Josef Semmler et Adriaan Verhulst, «Les statuts d'Adhalard de Corbie de l'an 822 », Le Moyen Âge, 17, 1962, p. 91-123 et p. 233-269 ; J.-C. HoCQUET, « Le pain, le vin et la juste mesure... », art. cit. Voir aussi le capitulaire de Villis : MONUMENTA GERMANIAE HiSTORICA, Capitularia Merowingica, op. cit., p. 84, chap. 9 : Volumus ut unusquisque iudex in suo ministerio mensuram modiorum, sextariorum (...) et corborum eo tenore habeant sicut et in palatio habemus. «Nous voulons que chaque juge ait dans sa juridiction une mesure des muids, des setiers et des corbes, de la même manière que nous en détenons une au palais ». 
d'application, au moins dans certains milieux. Cette question fondamentale pour les échanges doit encore être approfondie.

La quatrième règle est la reconnaissance d'une autorité susceptible d'assurer la police des échanges, c'est-à-dire d'imposer les monnaies, les poids et les mesures ainsi que de régler les conflits et les litiges nés autour des échanges ou à propos d'eux, lors de la réunion sur le marché, qu'il s'agisse de délits économiques ou de délits de droit commun, de fraude sur les quantités, de non-respect des procédures formelles ou encore de vols à l'étalage ou à l'arraché. La justice est alors rapide, violente et sans appel. Là encore, une enquête sur la répression des délits commis sur les marchés devrait être lancée. Elle trouverait sans doute nombre de récits aussi édifiants, si l'on peut dire, que ceux d'Hermann de Tournai concernant le comte Baudouin VII de Flandre au début du XII ${ }^{\mathrm{e}}$ siècle. Celui-ci exécutait sans procès et de la façon la plus spectaculaire, voire la plus cruelle, les hommes ayant troublé la paix des marchés ou n'ayant pas respecté les règles de l'échange. Un chevalier, coupable du vol de deux vaches, fut ainsi jeté dans une marmite d'eau bouillante encore revêtu de son armure. Une autre fois, neuf chevaliers furent pendus par le comte lui-même, toujours sans procès, pour avoir dérobé les bagages d'un marchand ${ }^{56}$. La stabilité de la propriété d'une part, la sûreté des échanges d'autre part, semblaient au comte des éléments suffisamment fondamentaux pour intervenir en cas de besoin avec la dernière brutalité.

Une fois ces éléments assurés, c'est-à-dire une fois instaurées les conditions matérielles et institutionnelles de l'échange, acheteurs et vendeurs peuvent se rencontrer et discuter des prix ou des moyens qu'ils jugent adéquats pour solder les échanges. Le marché, lieu physique de rencontre entre acheteurs et vendeurs, est aussi l'endroit où se forment les prix parce que les poids et les mesures, les monnaies, les rites et les sûretés existent. Ils permettent de constater l'existence de deux désirs convergents, celui de vendre et celui d'acheter et de les traduire en une évaluation. Dans le cas toutefois où un maximum est imposé, le prix fixé en dehors du marché est un prix administré. Il s'applique sur le lieu de l'échange mais ne s'y forme pas.

Après avoir procédé à ces rappels, nous allons maintenant nous arrêter sur les règles et les procédures semblant présider à la formation des prix, c'est-à-dire à l'attribution d'une valeur aux choses, qui accorde à la fois la volonté et le désir de l'acheteur et du vendeur et

\footnotetext{
56 - HERMAN OF TOURNAI, The restoration of the monastery of Saint Martin of Tournai, éd. par L. H. Nelson, Washington, Catholic University of America Press, 1996, p. 283.
} 
règle, de manière provisoire, l'antagonisme potentiel entre les deux parties de l'action d'acheter et de vendre, de demander et d'offrir.

\section{Règles de formation des prix et pratiques monastiques}

Qu'il existe des marchés, lieux physiques, où des valeurs sont mesurées et où se forment des prix avant que des objets ne soient échangés relève de ces évidences qu'il faut analyser et dont il est sans doute nécessaire de se méfier un peu. Les textes sont très peu diserts à ce propos, comme à chaque fois que l'on s'approche de ce que l'expérience commune considère comme allant de soi et n'ayant donc pas nécessairement besoin d'être dit, a fortiori écrit.

Les règles monastiques, telles qu'elles sont désormais étudiées, peuvent nous aider à comprendre un peu mieux ce qui se joue à ce propos : les moines se sont posés dès l'origine du cénobitisme la question de l'attitude convenable à observer à l'égard des lieux de l'échange. Leurs abbés doivent en effet gérer au mieux les biens terrestres de l'établissement dont le gouvernement leur est confié tout en favorisant le salut de l'âme de chacun de ses membres, dont la leur. Ils doivent donc composer avec les réalités de l'économie dont la question du profit, ainsi qu'avec les nécessités morales et spirituelles qui guident leur action ${ }^{57}$.

Valentina Toneatto vient précisément d'analyser ces questions en proposant une lecture d'un passage clé de la « règle du maître », l'une des sources d'inspiration de saint Benoît : le moment de fondation du monachisme occidental est un moment d'intense codification des pratiques, y compris économiques, ayant cours dans les cenobia. Un passage du texte de la règle du maître qu'elle commente en offre un saisissant exemple ${ }^{58}$. Le maître est interrogé

57 - V. TONEATTO, « Élites et rationalité économique... », art. cit.

58 - La règle du maître, éd. A. de Vogüé, Paris, Éd. du Cerf, 1964-1965, vol. 1, chap. 85, p. 221-

233 : Cum unaquaeque ars aliquod perfectum superuacuum usibus monasterii [...] abundauerit, interrogata qualitate pretii, quanti a saecularibus distrahi potest, a tanto infra numero nummorum et minori semper distrahatur pretio, ut agnoscatur in hac parte spiritales a saecularibus actorum distantia separari, cum non negotii causa, quae inimica est animae, lucrum supra iustitiam quaerant, sed etiam ab ipsa iustitia minus accipiendi praetii humanitate consentiant, ut non propter cupiditatem et auaritiam artes operari credantur, sed ne otio possit pascenda dignis sumptibus manus uacare [...]. Praetium uero acceptum abbati debere ab ipsis artificibus fideliter consignari. Quam deminutionem praetii extimatione abbatis artificibus debet constitui [...] . «Quand un métier quelconque aura un objet fabriqué en excédent par rapport aux besoins du monastère [...] après avoir enquêté sur le prix auquel les séculiers peuvent le vendre, on le vendra toujours pour une 
par son disciple sur ce qu'il convenait de faire des objets fabriqués au monastère et qui se trouveraient en surabondance (supervacua). Il répond qu'il faut les vendre, mais le faire à de certaines conditions.

Il s'agit ici de production artisanale, non de produits agricoles. Ainsi, il est normal, dans l'Italie du $\mathrm{VI}^{\mathrm{e}}$ siècle, de se poser ce genre de questions et tout aussi normal de considérer la vente comme une action allant de soi. La réponse donnée par le maître est, au demeurant, d'une très grande richesse et dévoile tout un lexique de l'échange, à la fois complexe et complet, et dont les mots-clés sont : supervacuum (surplus), usus, pretium, negotium, lucrum, iustitia, humanitas, cupiditas, avaritia, extimatio, emere. Ces mots s'articulent les uns par rapport aux autres et définissent une pratique de l'échange marchand destinée à habiter les consciences et à inspirer l'action : les moines ont médité sur ces textes et sur ces notions de façon ininterrompue durant tout le haut Moyen Âge et cela ne peut pas avoir été sans incidence dans la longue durée. Nous avons là, dans ces quelques lignes, une présentation du cadre cognitif à l'intérieur duquel se situent les pratiques économiques des moines du $\mathrm{VI}^{\mathrm{e}}$ siècle.

Le point saillant du propos, en ce qui nous concerne, touche aux prix. Ceux-ci sont fixés par l'abbé, après une enquête qui lui permet d'examiner ce qui se pratique alentour, à un niveau inférieur au prix du marché, mais pas sans rapport avec celui-ci. L'abbé ne doit pas en effet, par son intervention, provoquer leur baisse artificielle. Cela serait contraire à la charité

somme inférieure de monnaies et à un prix plus bas, afin que l'on sache que, dans ce domaine, les spirituels s'écartent des séculiers par leur manière d'agir. Ils ne cherchent pas, en effet, un gain qui dépasse la justice par des négociations qui sont ennemies de l'âme, mais même, ils consentent par amour du prochain à recevoir un prix inférieur à cette justice. Ainsi, l'on ne peut croire que c'est par cupidité et par avarice qu'ils exercentleurs métiers, mais pour qu'une main qui doit se sustenter dignement à ses dépens, ne puisse rester oisive [...] Le prix une fois reçu, les artisans doivent le remettre fidèlement à l'abbé. Cette réduction sur le prix doit être fixée aux artisans par une estimation de l'abbé [...]». Trad. A. de Vogüe, revue par V. Toneatto : V. TONEATTO, «Élites et rationalité économique... », art. cit. Voir aussi, sur ce passage : Giacomo TODESCHINI, I mercanti e il tempio. La società cristiana ed il circolo virtuoso della richezza fra Medioevo ed età moderna, Bologne, Il Mulino, 2002, p. 37-39. La teneur du texte est reprise, quoique abrégée, dans la règle de saint Benoît, au chapitre 57, J. Neufville et A. de Vogüe, éd. et trad. (Sources chrétiennes, 181-182183), La Règle de saint Benoît, Paris, 1972. Les deux sont repris dans la Concordia Regularum de Benoît d'Aniane au début du $\mathrm{IX}^{\mathrm{e}}$ siècle: Benedicti Anianensis Concordia Regularum, Pierre Bonnerue éd. , Turnhout, Brépols, 1999, II, p. 546-549 (Corpus Christianorum Continuatio Medievalis, CLXVIII B). 
et reviendrait à priver les laïcs de leur légitime profit et du fruit de leur travail en faisant se détourner d'eux les acheteurs. Cela serait aussi contraire à l'humanitas devant présider aux échanges dans une optique chrétienne. Il faut en revanche qu'il considère que lui-même n'a pas à tirer de son activité commerciale un gain excessif, étant entendu que les laïcs, eux, exagèrent toujours, et qu'ils cherchent pour leur part à tirer plus d'argent de leur activité artisanale ou commerciale qu'il n'est légitime de le faire.

Pour pouvoir procéder de la sorte, il est nécessaire de détenir une information sur les prix et donc s'être interrogé à la fois sur leur niveau et sur la qualité des objets en cause qu'il faut pouvoir comparer avant de déterminer un prix. L'abbé doit en effet juger de la signification de ceux-ci, ce qu'il faut entendre par la notion de qualitas pretii, ainsi que du rapport existant entre le prix du marché et la valeur effective de l'objet vendu : il fait alors preuve d'une qualité éminente et caractéristique de son rôle et de sa situation, en estimant ce qu'est la juste valeur de l'objet. Cela implique non seulement une connaissance des règles et des procédures du marché, mais aussi une capacité à juger de la qualité propre des objets résultant du travail manuel de moines ou d'autres hommes. On est en présence, ici, non pas d'un prix administré par une autorité extérieure au marché, mais d'un véritable prix d'expert.

On se trouve là, rappelle V. Toneatto, au cœur de ce que sont, de ce que doivent être les compétences des moines et, en particulier, celles de l'abbé. Ils doivent en effet savoir évaluer les choses, comme ils savent évaluer les actions et les pensées : tout cela est lié dans la très subtile construction qui organise le mode de pensée et d'action des moines bénédictins ou de leurs prédécesseurs. Ils savent juger de la valeur des pensées, qui sont bonnes ou mauvaises, concourent au salut ou à la damnation, de la même manière qu'ils jugent qu'une monnaie est bonne ou mauvaise ${ }^{59}$. Le moine est comme un changeur : par sa prière, il convertit les bonnes pensées en richesses spirituelles qui, accumulées, feront son salut. Or, les qualités mêmes qui permettent de juger de la valeur d'une pensée ou d'une action permettent d'en faire autant dans celle, matérielle, de la détermination de la valeur des choses.

Les jeux de métaphore et de transferts de sens existant autour de l'extimatio, de la probatio (capacité) et de la discretio (discernement) rendent possible l'établissement d'une identité, sinon paradoxale du moins inattendue, entre la sphère de l'échange marchand et celle de l'échange spirituel; entre l'échange établi par le monastère avec le monde qui l'entoure et celui qui s'établit entre les moines et Dieu. Enfin, l'estimation des choses doit, pour être efficace, rechercher la justice et non le lucre. C'est là l'essentiel : il y a un cadre de l'action

59 - V. TONEATTO, « Élites et rationalité économique... », art. cit. 
chrétienne en matière économique qui fonde une morale de l'action. L'abbé est celui qui sait estimer les valeurs et mobiliser cette compétence dans toutes les circonstances que sa charge lui impose. La justice (iustitia) est alors le résultat d'une négociation entre la necessitas du monastère et son utilitas. L'abbé enfin doit faire fructifier son patrimoine sans pour autant rechercher un profit excessif qui constituerait de l'avaritia : il doit faire gagner quelque chose au monastère, mais pas au-delà des besoins dont l'exacte définition constitue l'utilitas. Alors, et seulement si ces conditions sont réunies, le profit de la vente est converti, c'est-à-dire sanctifié, transformé en instrument de salut autant qu'en moyen de faire vivre dignement les moines. Les prix, ainsi, doivent être calculés en fonction des besoins de la communauté, comme des aléas des marchés.

La question de savoir si cette présentation peut être considérée comme valide durant tout le haut Moyen Âge doit encore être résolue. On peut cependant proposer l'hypothèse selon laquelle les réflexions du maître auraient structuré la réflexion et la pratique des moines occidentaux durant tout la période et que les notions qu'elle utilise sont peu ou prou connues de tous les gestionnaires : la présence du texte de la «Règle du Maître » dans la Concordia Regularum de Benoît d'Aniane est un indice en ce sens.

\section{Calculer, estimer, mesurer}

Même si la Règle du Maître est peu connue au IX ${ }^{\mathrm{e}}$ siècle, la méditation sur des textes de même nature, traitant de sujets proches, où l'action et la contemplation se croisent et s'entremêlent, est permanente dans le monde monastique. Les comportements y sont façonnés par ces normes qui, tout en concernant d'abord le salut de l'âme, amènent à définir ce qu'est le «bien agir », l'agir chrétien, en matière économique. Il n'est alors pas indispensable de développer un discours autonome, une doctrine, sur la vie économique, parce que la réflexion sur les catégories posées d'entrée de jeu par les Pères de l'Église et par les législateurs de la génération de saint Benoît fournissent un arrière-plan à la fois spirituel et théorique aux praticiens de la gestion que sont les administrateurs de la vie monastique. La capacité à estimer les actions, les pensées et les choses constitue le caractère distinctif du bon abbé qui est et doit être un bon intendant aussi bien pour les âmes que pour les biens du monastère ${ }^{60}$. L'important est que, d'une part, il existe un marché sur lequel s'écoulent des produits agricoles ou artisanaux. Les prix s'y forment sans intervention extérieure et il est possible de

60 - V. TONEAtTo, « Marchands et banquiers du Seigneur... », op. cit., p. 301 sq. et 529-541. 
constater l'existence d'une sorte de prix moyen. L'abbé intervient ensuite et fixe, par un acte arbitraire censé établir son humanitas ainsi que sa capacité de gestionnaire, un autre prix que celui-ci qui préexiste, comme une condition nécessaire.

La pertinence du texte du maître et la possibilité d'y recourir reposent toutefois sur un présupposé matériel dont la réalisation permet de poser la question de la vente : l'existence de surplus de production, faute de quoi ces réflexions ne feraient pas sens. Si, dans l'Italie d'avant les guerres lombardes, un certain niveau d'échanges locaux est tout à fait envisageable, il n'est pas illégitime d'avoir des doutes sur ce qui se produit ensuite, durant le $\mathrm{VII}^{\mathrm{e}}$ siècle et la plus grande partie du VIII siècle. Nous savons cependant, par le biais des coutumes monastiques, qu'il y a toujours eu des ateliers dans les monastères, que ce soit à Saint-Gall ou dans des établissements plus modestes comme par exemple Murbach, où, en 816 , les Statuts indiquent la présence d'ateliers de foulons, de tailleurs, de cordonniers et que ceux-ci sont placés à l'intérieur de la clôture ${ }^{61}$. Il en est de même à Bobbio, où les statuts de l'abbé Wala mentionnent des ateliers de fabrication d'armes, toujours dans l'enceinte du monastère ${ }^{62}$. À Corbie, la liste des provendarii prévoit la présence d'un certain nombre d'artisans, une vingtaine, spécialisés ou non ${ }^{63}$. Et, de fait, saint Benoît a insisté dans la règle pour que tous les métiers nécessaires au monastère y soient représentés. La situation prévue par le maître, à savoir l'existence de supervacua, a toute chance d'avoir existé, du moins dans les plus riches et les mieux organisés des monastères. Les monastères sont normalement en situation d'offrir des biens sur les marchés, selon une logique de l'autarcie qu'il ne faut pas réduire à une volonté d'autoconsommation mais qui sous-entend que les surplus soient vendus et que le produit de la vente serve à couvrir les besoins en biens non disponibles sur place ${ }^{64}$.

Il faut cependant nuancer. C'est essentiellement là une possibilité et, dans bien des cas, elle ne peut pas se réaliser: les gestionnaires monastiques sont souvent rattrapés par une

61 - Jean-Pierre DEVROEY, Économie rurale et société dans l'Europe franque (VI'-IXe siècles), Paris, Belin, 2003, p. 132.

62 - Codice diplomatico del monastero di S. Colombano di Bobbio fino all'anno 1208, éd. par C. Cipolla, Rome, Tip. del Senato, 1918, p. 136-141.

63 - Statuta seu Brevia Adalhardi abbatis Corbeinsis, éd. par J. Semmler, Siegburg, Franz Schmitt, 1963, p. 355-422, particulièrement p. 367. On dénombre des couturiers (ou tailleurs), des palefreniers, un foulon, des orfèvres, des forgerons, un «parcheminier», des fabricants de boucliers, des fabricants d'armes. Il n'est donc pas structurellement impossible que le monastère, à l'occasion, produise des surplus et soit en position de vendre.

64 - J.-P. Devroey, Économie rurale..., op. cit., p. 174 ; Id., Puissants et misérables..., p. 585-612. 
réalité où les surplus sont rares et où les occasions de vendre le sont aussi. Les monastères sont fréquemment soumis à des difficultés économiques de tous ordres. Ainsi, par exemple, Loup de Ferrières, dans les lettres qu'il adresse à ses amis, se plaint de ce que la perte de sa dépendance de Saint-Josse prive tout à fait le monastère et ses moines de ses sources normales d'approvisionnement en vêtements et en nourriture ${ }^{65}$. Il ne peut pas faire face non plus à l'ensemble de ses charges qui comprennent, outre l'entretien des moines et l'accueil des hôtes, des dons annuels au roi. Il doit régulièrement acheter du blé et les frères sont fréquemment contraints de se nourrir de légumes. Ils sont obligés de porter des vêtements raccommodés. En 845, lors d'une famine, Loup a même dû vendre quelques vases sacrés pour nourrir la communauté et sa familia. Il n'emprunte pas mais est contraint de déthésauriser et son trésor n'est pas sous forme monétaire.

Loup n'exagère pas et ne noircit pas à dessein la situation dans des lettres où il s'adresse à ses moines. Bien qu'il soit à la tête d'un grand monastère, et que lui-même soit proche du souverain, son établissement connaît de réelles difficultés qui le mettent dans une situation délicate. Sur le marché des céréales, en tout cas, il est demandeur, rarement offreur, et l'absence de stocks est cause de tracas pour sa communauté. Alors que Cluny, aux $\mathrm{XI}^{\mathrm{e}}$ et $\mathrm{XII}^{\mathrm{e}}$ siècles, convertit immédiatement en argent les redevances de ses dépendances lointaines et que ses gestionnaires savent bien que les exteriora, les biens que le monastère ne produit pas, doivent être achetés, les moines de Ferrières vivent, ou feignent de vivre dans un monde où l'échange marchand est réduit à sa plus simple expression et où en tout cas ils ne veulent intervenir que comme consommateurs de ce qui est produit sur leurs domaines ou comme acheteurs de denrées ${ }^{66}$. L'équation de Loup n'intègre pas la vente des surplus et la constitution de réserves alimentaires ou monétaires.

Cette situation n'empêche pas cependant la présence, même chez Loup de Ferrières, de ces compétences particulières, applicables aussi au domaine de l'économique qui caractérisent les moines bénédictins et que $\mathrm{V}$. Toneatto résume sous le vocable de virtuosité, une virtuosité qui s'applique à l'ensemble des domaines où l'évaluation a cours, qu'il s'agisse des pensées ou des choses impliquées ou non dans les échanges.

65 - Loup DE Ferrieres, Correspondance, éd. par L. Levillain, Paris, Champion, 1927, t. 1, p. 181, 1. $n^{\circ}$ 43. Sur les embarras financiers de Loup, ibid., p. 191, lettre $n^{\circ} 45$.

66 - Georges DuBY, « Le budget de l'abbaye de Cluny entre 1080 et 1155 . Économie domaniale et économie monétaire », Annales ESC, 7-2, 1952, p. 155-171 ; Alain GUERREAU, « Douze doyennés clunisiens au milieu du XII ${ }^{\mathrm{e}}$ siècle », Annales de Bourgogne, 52-2/3,1980, p. 83-128. 
La virtuosité monastique est admirablement illustrée dans un certain nombre d'exemples. J'en retiendrai deux qui mettent l'un et l'autre en cause le même personnage, Adhalard, membre de la famille carolingienne, abbé de Corbie et missus en Italie à la fin du règne de Charlemagne ${ }^{67}$. Les statuts qu'il rédigea vers 822 et qui devaient servir aussi bien de texte d'application de la règle que de guide à vocation pédagogique pour ses successeurs en sont un exemple tout à fait remarquable. Adalhard est un homme qui aime les chiffres, les dénombrements et les proportions. Il compte, additionne et soustrait, mais surtout multiplie et divise d'une manière qui n'a rien d'élémentaire. Le chapitre qu'il consacre à l'annone apparaît en ce sens comme un modèle où le mot ratio, au sens de proportion, semble structurer l'ensemble du raisonnement ${ }^{68}$. Adalhard se livre dans ces pages à une série de calculs destinés à établir de quelle quantité de grains d'épeautre le monastère avait besoin pour couvrir l'ensemble de ses besoins alimentaires. Il fallait, pour que la démarche ait une signification et que les calculs aient quelque validité, qu'il ait une bonne connaissance des quantités disponibles ainsi que de la qualité particulière des grains, différente à chaque récolte : le même volume de grain ne donne pas toujours la même quantité de farine... Il ne sait pas, en revanche, avec certitude quel est le nombre de bouches à nourrir : les moines, les hôtes et les pauvres ne sont pas en nombre fixe et stable. Adalhard estime qu'ils ne sont jamais moins de 300 et sont très souvent plus de 400. Toute la difficulté de l'exercice auquel il se livre afin de prévoir, en bon gestionnaire, l'organisation de l'approvisionnement vient de cette série d'incertitudes portant aussi bien sur la qualité des grains dont il dispose que sur le nombre effectif de bouches qu'il doit nourrir. S'il se trompe en excès, c'est-à-dire s'il fait cuire trop de pains, le surplus sera donné. Donné, non vendu, mais s'agissant d'aliments et, en dernière analyse, de la survie des bénéficiaires des dons, cela ne doit pas étonner. Les produits alimentaires ont bien évidemment un statut différent de ceux provenant de l'activité artisanale. Les calculs auxquels se livre Adalhard ont longtemps passé pour être embrouillés et approximatifs, ce qu'ils ne sont pas, mais sont caractéristiques du personnage. Il aime à la fois les chiffres et les calculs d'équivalence. Il a une certaine maîtrise de la règle de trois, même s'il ne peut pas la poser. Par exemple, sachant que 4 muids de grain permettent de fabriquer 120 pains, il peut établir combien il faut de muids pour en faire 300 et passer pour

\footnotetext{
67 - Brigitte KASten, Adalhard von Corbie. Die Biographie eines karolingischen Politikers und Klostervorstehers, Düsseldorf, Droste, 1985.

68 - Statuta seu Brevia Adalhardi..., op. cit., p. 375-379. Voir J.-C. HoCQUET, « Le pain, le vin et la juste mesure... », art. cit. ; J.-P. DEVROEY, Puissants et misérables..., op. cit., p. 607.
} 
ce faire d'une unité de mesure à une autre. Le muid est en effet un sous-multiple de la corbe, qui vaut 12 muids, laquelle représenterait la quantité de grains que peut déplacer une charrette ${ }^{69}$. Bref, il se livre à des calculs assez compliqués qui ont mystifié savants et éditeurs mais qui montrent un goût évident, et partagé par les membres de l'élite culturelle carolingienne, pour la mesure des choses, les calculs d'équivalence et les conversions. Les dispositions d'Adalhard, qui sont celles qu'Alcuin développait chez ses disciples, sont également celles dont les règles du maître et de saint Benoît prévoient l'existence chez les abbés, la capacité d'estimer allant de pair avec celle de calculer ${ }^{70}$. Le texte d'Adalhard apparaît bel et bien comme un exercice de virtuosité dans lequel il montre, peut-être avec quelque complaisance, l'étendue de ses capacités dans l'exercice complexe qu'est celui de l'estimation et de la mesure. Il mobilise ces capacités en tant que de besoin.

Nous disposons en effet, à propos d'Adalhard, d'un autre exemple beaucoup plus concret encore que celui fourni par les Statuts, dans lequel l'abbé de Corbie mesure des choses, en l'occurrence des terres, et en établit la valeur. Il s'agit d'un texte datant de 813 , un échange entre deux monastères, Saint-Sauveur de Brescia et Nonantola auquel il a été demandé à Adalhard de présider en tant que missus de Charlemagne ${ }^{71}$. Il a longtemps été compris à contresens, du fait de la mauvaise qualité de l'édition disponible et a été utilisé comme une preuve de l'arbitraire des hommes du haut Moyen Âge dans la détermination de la valeur des terres ${ }^{72}$.

69 - J.-C. HOCQUET, « Le pain, le vin et la juste mesure... », art. cit.

70 - Mayke De JONG, « From scolastici to scioli. Alcuin and the formation of an intellectual elite »,

in L. Houwen et A. MACDONALD (dir.), Alcuin of York: Scholar at the Carolingian court, Groningue, Forsten Pub., 1998, p. 45-58.

71 - Pour ce qui suit, voir l'article essentiel de F. BOUGARD, « Adalhard de Corbie entre Nonantola et Brescia (813) : commutatio, gestion des biens monastiques et marché de la terre », in E. CuOzzO et al. (dir.), Puer Apuliae. Mélanges offerts à Jean-Marie Martin, Paris, Association des amis du centre d'histoire et civilisation de Byzance, 2008, p.51-68. F. Bougard donne une excellente traduction française du texte d'autant plus utile qu'elle évite toute ambiguïté en prenant des partis qui rendent compte du sens du texte et de la polysémie de certains mots comme gratia qui est l'une de ses caractéristiques. F. Bougard utilise ici la traduction comme un outil heuristique dont, par l'exemple, il montre la puissance. Ce texte, longtemps inutilisable du fait de l'édition défectueuse de Porro Lambertenghi, a été rendu accessible par une édition récente: G. Feo et al. (dir.), Chartae Latinae Antiquiores, Italy LX, Modena, Nonantola, I, Zurich, Urs Graf Verl., 2008, ${ }^{\circ} 29$.

72 - Voir, en dernier lieu, Chris WiCKHAM, « Conclusions », in L. FELler et C. WiCKHAM (dir.), Le marché de la terre au Moyen Âge, Rome, École française de Rome, 2005, p. 625-642 
Dans le cadre d'un échange où interviennent des établissements monastiques, il faut, selon la loi, que chacun d'entre eux tire un profit de l'opération, que ce soit en valeur du capital en ou en montant des revenus. Adalhard s'efforce de montrer, en comparant les valeurs des différentes terres en cause, que l'opération de remembrement apportera un bénéfice en capital aux deux abbayes et que leurs revenus s'en trouveront effectivement améliorés. Or, l'un des problèmes difficiles du texte est l'énorme disparité de la valeur des terres concernées : un jugère situé près de Brescia vaut au moins 3 sous, tandis qu'il ne vaut que 8 deniers près de Nonantola.

On retenait habituellement du texte que les prix n'avaient pas de sens économique mais qu'ils étaient fixés en fonction de critères familiaux, sociaux, politiques et que, en bref, ils ne signifiaient pas grand-chose de compréhensible pour nous. La traduction et le commentaire de F. Bougard permettent d'établir que, au contraire, seule une connaissance très fine des conditions concrètes de la vie économique expliquait le prix et son niveau. En effet, des critères permettant d'établir la valeur des terres ressortent nettement de la lecture du texte : la distance des parcelles considérées d'avec les centres de gestion monastique, la présence de bâtiments d'exploitation, le rendement des terres en grains de différentes qualités, le froment gaulois dans un cas, le siligo, variété padane dans l'autre ${ }^{73}$. Enfin, la proximité de voies de communication est elle aussi prise en compte: la valeur du bien inclut donc des considérations sur le coût du transport.

Pour parvenir à ce résultat, une commission d'hommes particulièrement compétents a été rassemblée et a enquêté sur place, ce qui renvoie bien entendu aux procédures monastiques les plus anciennes, telles que nous les avons déjà vues définies dans la règle du maître. Les conclusions de la commission sont que les lieux ne sont pas équivalents, le produit, entendu ici au sens de profit, de l'un et de l'autre étant différent mais que le revenu tiré de chacune des terres sera accru du fait de la diminution de la distance avec le centre ainsi que du remembrement qui sera opéré. On s'attend donc à ce que les revenus des deux parties s'accroissent. De même, l'enquête sur les prix pratiqués implique ici une procédure qui, sortant des règles propres de l'échange marchand, fait intervenir le « pouvoir discrétionnaire » du président de la commission, l'abbé Adalhard. Celui-ci, qui sait estimer, sait aussi dans quelle mesure il peut intervenir sur le prix des terres, tout comme il le fait sur celui des choses qu'il produit en surplus et vend au marché. Pour fixer le prix de la terre dans le cadre de cet échange, la connaissance du prix du marché est nécessaire mais pas suffisante. Le jugement

73 - F. BOUGARD, « Adhalard de Corbie... », art. cit., p. 58, § 4. 
de l'acteur qu'est l'abbé est, lui aussi, indispensable et lui permet de n'être pas prisonnier de prix déjà formés. Connaissant l'utilité des choses, mesurées ici par le rendement de la terre, il avance aussi d'autres chiffres reposant sur l'établissement de proportions. Ainsi, la terre proche de Brescia et qui appartient à Nonantola, vaut trois fois le prix de l'autre qui, située près de Nonantola appartient à Brescia. Il faut, pour arriver à cela, laisser faire l'abbé qui augmente un peu le prix de marché de l'une des terres et diminue celui de l'autre ${ }^{74}$. La manipulation du prix, opérée par l'abbé afin de construire une proportion aisément calculable est admise et est en fait conforme aux prescriptions de la règle du maître : l'abbé peut intervenir sur les prix et les modifier. Il n'est en aucune manière prisonnier du marché. L'inégalité des surfaces est d'autre part compensée par la présence de bâtiments en dur. Ceux construits sur la terre proche de Brescia valent au total 15 livres, ce qui porte la valeur commerciale de ce domaine d'une superficie de 110 jugères à 31 livres. Pour la terre située près de Nonantola, elle porte des bâtiments ayant une valeur de 100 sous ( 5 livres), mais est de meilleur rapport. La compensation de la valeur en capital se fait en ajoutant trois églises à ce domaine.

Le jeu arithmétique auquel se livre Adhalard est assez sophistiqué et repose sur un principe : toute chose peut être mesurée et a une valeur. Les simplifications faites pour parvenir à des comparaisons terme à terme sont parfaitement légitimes : la terre située près de la ville vaut finalement trois fois plus que celle située près du monastère, en pleine campagne, bien que celle-ci soit d'un meilleur rapport. On entrevoit, par-derrière ces supputations et ces estimations, la présence d'un marché du grain comme celle d'un marché de la terre ainsi qu'une connaissance du coût du transport. La présence d'une ville, même de petite dimension, est aussi un facteur que l'on ne peut considérer comme neutre ou indifférent. Bref, ce texte place l'abbé de Corbie dans la fonction qui est bien la sienne : il est celui qui détermine et fixe la valeur des choses parce qu'il est en mesure d'intégrer l'ensemble des données disponibles et constatables. Il détermine ce que l'on peut appeler un prix d'expert. Mais les arguments d'Adalhard ne prennent sens qu'une fois admis qu'il existe aussi à l'œuvre des mécanismes autonomes de formation des prix reposant sur des critères objectifs et, eux aussi, mesurables.

\footnotetext{
74 - Je m'éloigne ici de l'interprétation de F. Bougard qui comprend le mot pertinere ad comme un rapport de proximité géographique alors qu'il s'agit, à mon sens d'un rapport de propriété : la terra (...) que ad Brexia pertinet est celle dont le monastère non nommé de Brescia est propriétaire et c'est elle qui, logiquement, a une valeur d'échange trois fois supérieure à celle située près de Nonantola.
} 
Enfin, l'intervention de l'abbé à la fois estimator et expert corrige les résultats de l'enquête en manipulant les valeurs et en intégrant les éléments non productifs, à savoir des bâtiments et des églises et donc en réintroduisant sa propre estimation de la valeur des choses ainsi que de leur utilité. Il le fait conformément aux principes juridiques mais aussi conformément aux aptitudes particulières développées chez lui par l'habitude de la conversion et de la détermination des proportions, ce qui lui permet de parvenir à un prix que l'on peut qualifier de juste, c'est-à-dire qui rende justice aux deux parties concernées en accroissant la valeur de leur capital et le montant de leurs revenus. L'abbé prend acte des mécanismes économiques à l'œuvre en dehors de lui, mais ne renonce pas à intervenir de façon à ajuster les valeurs, sans avoir à en donner la raison, parce qu'en toutes choses, il recherche d'abord la justice.

\section{Famines et disettes}

Ces mécanismes économiques sont parfois mis au jour et éclairés lorsque famines et disettes révèlent, parfois de façon crue, le fonctionnement concret du système des échanges ainsi que les déficiences ou les carences du système de production ${ }^{75}$.

75 - Voir, en attendant la publication du volume de recherche collectives du groupe dont il dirige l'activité avec Monique Bourin, la mise au point de François MENANT, «Crisis de subsistencia y crisis agrarias en la Edad Media: algunas reflexiones previas », in H. Oliva HerRer et P. BENITO (dir.), Crisis de subsistencia y crisis agrarias en la Edad Media, Séville, Universidad de Sevilla, 2007, p. 17-60. On attend avec impatience l'ouvrage que Pere Benito prépare sur les famines médiévales prises dans leur ensemble. Voir, en dernier lieu, Pere BENITO, « Retour au Toulousain. Famines graves, exode et persistance de la famine en Catalogne à l'époque du comte Raimond Borell », in C. DENJEAN (dir.), Sources sérielles et prix au Moyen Âge. Travaux offerts à Maurice Berthe, Toulouse, CNRS-université de Toulouse-Le Mirail, 2009, p. 43-80. La mobilisation des thèses de A. Sen a considérablement contribué à faire repartir une réflexion bloquée par les apories construites et laissées en l'état par le débat des années 1960-1970. Voir, pour une approche de la question: Trevor H. Aston et Charles H. E. Philpin (dir.), The Brenner debate: Agrarian class structure and economic development in pre-industrial Europe, Cambridge, Cambridge University Press, 1985. Il a fallu attendre les travaux de William Jordan pour que l'on commence à sortir, en ce qui concerne la crise du bas Moyen Âge, des termes du débat imposé par les néo-malthusiens : William C. JORDAN, The great famine: Northern Europe in the early fourteenth century, Princeton, Princeton University Press, 1996. Voir, surtout, l'ouvrage fondamental pour notre propos de Amartya SEN, Poverty and famines: An essay on entitlement and deprivation, Oxford, Clarendon Press, 1981. 
L'époque carolingienne a connu de nombreuses famines dont certaines ont été particulièrement meurtrières. Les souverains carolingiens ont produit à leur sujet un certain nombre de textes normatifs dont le propos est autant idéologique et moral qu'économique. Les allusions faites aux difficultés des temps sont en effet nombreuses : elles ne se rapportent pas nécessairement à une famine en particulier, mais bien plutôt à une atmosphère générale, marquée par des guerres et des difficultés politiques, parfois extrêmement rudes et que les clercs résument sous le vocable de tribulations, sans qu'il soit toujours possible de dire ce qui s'est passé ${ }^{76}$. Par exemple, le capitulaire de Thionville énumère toute une série de calamités contre lesquelles il faut prendre des mesures immédiates au premier rang desquelles la prière, sans que le roi ait besoin de rien ordonner ${ }^{77}$. La situation de l'année semblant l'exiger, des mesures limitées sont imposées pour lutter contre la famis inopia, la disette : ne pas vendre trop cher son grain et ne pas exporter de blé hors de l'empire.

En temps de famine, cependant, les céréales n'ont littéralement pas de prix parce qu'il n'y a pas d'offre, la stérilité de la nature rendant impossible tout échange. C'est la situation que constatent, du moins apparemment, les Annales Mosellani pour l'année 793. La pénurie d'aliments contraint de recourir à des nourritures immondes; des affaires de cannibalisme se produisent, les hommes se mangeant entre eux, les frères dévorant les frères et les femmes leurs enfants ${ }^{78}$. Un phénomène que l'on s'explique fort mal prend ensuite place en 794 ,

76 - Marcelo CANDIDO DA SILVA, L'« économie morale » carolingienne (fin VIII -début IXe siècle), à paraître.

77 - Monumenta Germaniae Historica, Leges, t. IV, Karoli Magni Capitularia, éd. par A. Boretius, Berlin, Weidmann, 1824, n 44, p. 122-123 (a. 805) : De hoc si evenerit fames, clades, pestilentia, inaequalitas aeris vel alia qualiscumque tribulatio, ut non expectetur edictum nostrum, sed statim depraecetur Dei misericordia. « Si survient une famine, une épidémie, une pestilence, un désordre météorologique ou quelque autre accident, que l'on attende pas notre édit mais que sur le champ l'on se mette à implorer la miséricorde divine ».

78 - Monumenta Germaniae Historica, Scriptores, t. XVI, Annales Mosellani, Hannovre, Hahn, 1869, p. 498, n 23. Famis vero, quae anno priori caepit, in tantum excrevit, ut non solum alias immundicias, verum etiam, peccatis nostris exigentibus, ut homines homines, fratres fratres ac matres filios comedere coegit. Ostensa autem eodem anno in ipso regno per diversa loca vemo tempore falsa annona per campos et silvas atque paludes innummera multitudo, quam videre et tangere poterant, sed comedere nullus. «La famine, qui a commencé l'année d'avant, devient si grande que non seulement elle contraint à manger des nourritures immondes, mais que, en plus, conséquence de nos péchés, elle fait que des hommes mangent des hommes, les frères des frères, et les mères leurs enfants. On vit la même année, dans le royaume, par divers lieux, de fausses récoltes 
toujours selon le même texte : les champs, les forêts, les sylves et les paluds semblent fertiles, mais ce qu'ils produisent est impropre à la consommation. C'est donc ici un défaut de production qui est clairement mis en cause. La réponse de Charlemagne en 794 ne correspond pourtant pas à cette constatation. Il intervient en effet sur les prix et sur les règles de l'échange en établissant un maximum des prix des céréales, qui n'est pas occasionnel mais est présenté comme absolu : quelles que soient les circonstances, que l'on soit en temps d'abondance ou en temps de disette, les prix ne devraient pas dépasser le niveau qu'il fixe, déterminé par rapport à une quantité stable, le muid public, et défini grâce à la nouvelle monnaie ${ }^{79}$. L'existence d'un couple d'opposition est ici constatée, celui de l'abundantia et de la caritas. L'abondance frumentaire s'oppose au renchérissement des prix : la crise de 794 peut sans doute être considérée d'abord comme ayant donné lieu à une hausse très violente. Établir des règles claires à l'échange qui assurent la stabilité des prix et définissent une fois pour toutes la valeur des choses est faire œuvre de justice. Le montant exact de ces maxima autorisés importe moins ici que l'obligation faite de recourir au muid public et au denier et que l'institution d'une proportion entre les différentes valeurs des céréales panifiables proposées sur le marché. Il est en effet très peu probable, et ît est même impensable, que Charlemagne ait réellement cherché à imposer un maximum valable pour tout l'empire. Le marché sur des territoires aussi vastes ne peut pas fonctionner de façon homogène et les conditions de formation des prix ne peuvent pas être identiques de la plaine du Po au cours de l'Elbe ou de l'Èbre. D'autre part, on ne voit pas très bien comment Charlemagne aurait pu rassembler des données sur les prix dans l'ensemble de l'empire, si tant est qu'il eût estimé utile de collationner des renseignements de ce type. En revanche, l'effet de la mesure est d'établir des

dans les champs, les sylves et les marais : on voyait et touchait une immense quantité de denrées mais on ne pouvait rien en manger $\gg$. . Voir sur ce passage et ses obscurités: Vincent VANDENBERGH, "'Fames facta est ut homo hominem comederet' : l'Occident médiéval face au cannibalisme de survie $\left(\mathrm{V}^{\mathrm{e}}-\mathrm{XI}^{\mathrm{e}}\right.$ siècle) », Revue belge de philologie et d'histoire, 86-2, 2008, p. 217 272, ici p. 243-244. Voir également Jean-Pierre DEVROEY, «Dîme et économie des campagnes à l'époque carolingienne », in R. VIADER (dir.), La dîme dans l'Europe médiévale et moderne, Toulouse, Presses universitaires du Mirail, 2010, p. 38-63, ici p. 48 et 50.

79 - Monumenta Germaniae Historica, Karoli Magni Capitularia, op. cit., n 23, p. 73-74, § 4 : ut nunquam carius vendat annonam sive tempore abundantiae sive tempore caritatis, quam modium publicum et noviter statutum, de modio avena denario duo... «Que personne ne vende le grain, que ce soit en temps d'abondance ou en temps de cherté, en mesurant à l'aide du muid public nouvellement établi, plu scher que deux deniers le muid d'avoine »... 
proportions entre les prix des différentes céréales d'une part et entre le marché et ce que l'on pourrait appeler le prix d'intervention des autorités publiques.

Maximum des prix dans les capitulaires de Nimègue et de Francfort

\begin{tabular}{|l|l|l|l|}
\hline & $\begin{array}{l}\text { Vendeurs « ordinaires » } \\
\text { Francfort (794) }\end{array}$ & $\begin{array}{l}\text { Annona publica, si } \\
\text { vendue } \\
\text { Francfort (794) }\end{array}$ & Nimègue (806) \\
\hline Avoine 1 muid & $2 \mathrm{~d}$ & $0,5 \mathrm{~d}$ & $2 \mathrm{~d}$ \\
\hline Orge 1 muid & $2 \mathrm{~d}$ & $1 \mathrm{~d}$ & $3 \mathrm{~d}$ \\
\hline Épeautre & - & - & $3 \mathrm{~d}$ \\
\hline Seigle 1 muid & $3 \mathrm{~d}$ & $2 \mathrm{~d}$ & $4 \mathrm{~d}$ \\
\hline Froment 1 muid & $4 \mathrm{~d}$ & $3 \mathrm{~d}$ & $6 \mathrm{~d}$ \\
\hline
\end{tabular}

Les prix proposés sont des maxima dont la fonction est surtout d'établir des proportions entre les valeurs des différents grains. Les autorités publiques peuvent intervenir en vendant des grains appartenant au souverain à un prix inférieur à celui pratiqué sur les marchés.

Il est en effet prévu que les officiers publics vendent les céréales stockées dans les greniers impériaux : ils le font à des prix très inférieurs au prix du marché, les céréales secondaires subissant une baisse beaucoup plus drastique, au demeurant, que le froment ou le seigle. Des mesures de cette nature sont réitérées à Nimègue en 806 et l'on constate que, d'une part, les prix ont monté et que, d'autre part, les proportions entre les valeurs ont, elles aussi bougé.

L'intervention des greniers royaux en 794 est le signe manifeste du refus du roi de participer à la spéculation à la hausse ou de laisser faire celle-ci sans réagir : en jetant ses réserves sur le marché, il élargit l'offre ou plutôt fait une nouvelle offre, plus basse. Les mécanismes élémentaires de la hausse sont parfaitement connus et identifiés : le capitulaire de Nimègue, promulgué en 806 , les décrit avec beaucoup de simplicité en les rangeant dans des catégories morales, celles de turpe lucrum et d'avaritia, à quoi s'oppose le negotium ${ }^{80}$. Le texte procède en effet à plusieurs rappels qui doivent fonder la vie économique comme la vie morale. D'abord, les trésors de l'Église ne doivent pas être vendus (§ 4) et particulièrement pas aux marchands juifs. La propriété royale doit être sauvegardée (§6) et les mendiants protégés $(\S 8)$. Le capitulaire développe ensuite une réflexion morale élémentaire en définissant l'usure, la cupiditas, l'avaritia, le turpe lucrum et le foenus, qui seul apparaît

${ }^{80}-$ Ibid., $\mathrm{n}^{\circ} 46$, p. 132. 
comme légitime ${ }^{81}$. L'exemple donné pour éclairer la définition du turpe lucrum est remarquablement éclairant : acheter au moment de la moisson ou de la vendange au-delà de ses besoins et attendre pour revendre que le prix monte, ce qui arrive immanquablement à partir du mois de janvier, produit un gain honteux ${ }^{82}$. Acheter pour couvrir ses besoins et éventuellement redistribuer à autrui relève du negotium, et le bénéfice limité que l'on peut en tirer s'appelle foenus.

Il est notable que cette définition purement morale ne s'accompagne d'aucune sanction pénale : ce n'est pas un délit, mais un dévoiement dans la recherche d'un comportement véritablement chrétien qui permette de s'occuper de la production et de l'échange tout en faisant son salut: ce type de comportement a été qualifié par G. Todeschini d'éthicoéconomique, formule particulièrement bien choisie en ceci qu'elle permet d'établir la synthèse entre deux pôles normalement antinomiques du comportement économique, rationnel en finalité ou en moyens ${ }^{83}$. Ce comportement est lié à une organisation concrète et physique du marché, qui passe par le recours aux mesures et aux monnaies. Il implique également une dénonciation des comportements spéculatifs, dont nous avons de nombreux exemples pour la période.

Le passage du concile de 829 auquel il a été fait allusion plus haut comporte une description circonstanciée du mécanisme de la spéculation et du prêt à intérêt et donne un

81 - Pour une définition de ces catégories, dans le contexte de la génération successive : Gaëlle , «Cupiditas, avaritia, turpe lucrum. Discours économique et morale chrétienne chez Hincmar de Reims (845-882) », in J.-P. DeVRoey, L. Feller et R. LE JAN (dir.) Les élites et la richesse..., op. cit., p. 97-112.

82 - Monumenta Germaniae Historica, Karoli Magni Capitularia, op. cit., n 46, p. 132 : Quicumque enim tempore messis vel tempore vindemiae non necessitate sed propter cupiditatem comparat annonam aut vinum, verbi gratia de duobus denariis comparat modium unum et servat usque dum iterum venundare possit contra dinarios quatuor aut sex seu amplius, hoc turpe lucrum dicimus ; si autem propter necessitatem comparat, ut sibi habeat et aliis tribuat, negotium dicimus. «Quiconque achète au temps de la moisson ou de la vendange mais à cause de sa cupidité du grain ou du vin, et qu'il achète, par exemple, un muid pour deux deniers et qu'il le conserve jusqu'à ce qu'il puisse le vendre contre quatre ou six deniers, voire davantage, cela nous l'appelons un gain honteux. S'il achète pour ses besoins, qu'il le garde pour lui et en fournit à d'autres, cela nous l'appelons négoce ».

83 - Voir, pour une définition de ce concept, G. TODESCHINI, Richesse franciscaine..., op. cit. M. Weber, Économie et société, op. cit., p. 396-397. J.-P. DeVroey, Puissants et misérables..., op. cit., p. 605-608. 
exemple théorique mais particulièrement saisissant. Celui qui ne peut payer comptant le blé qu'il achète s'engage à le rembourser l'année suivante en nature en estimant non pas le volume mais la valeur du blé à ce moment. Autrement dit, s'il achète un muid valant 2 deniers en 829 , il remboursera en 830 non pas un muid mais ce que 2 deniers permettront d'acheter à ce moment-là. Or, on s'attend à ce que les mauvaises années ne s'enchaînent pas : le pari du prêteur est que, la récolte de 830 étant bonne, les prix auront baissé et que le débiteur sera contraint de donner une quantité de blé bien plus importante que ce qu'il aura obtenu en 829 . Le mécanisme est simple : c'est une spéculation à la baisse. Il implique une prise de risque réelle de la part du prêteur, et qui a toute chance d'être très coûteuse pour l'emprunteur. Les acteurs ici jouent sur un grand nombre de facteurs: les quantités, l'évaluation de la marchandise, la variation des prix, l'alternance entre paiements en nature et paiements en argent. L'argent est alors ici essentiellement un instrument de mesure de la valeur et n'est pas destiné à servir de medium de l'échange, puisque c'est l'absence d'argent ou de tout titre permettant d'accéder au marché des céréales qui provoque la mise en route du mécanisme.

Cela renvoie à une économie où quelques-unes des observations faites par A. Sen sont également valides :

- Les mesures prises par Charlemagne ou les pères du concile de 829 ne font sens que s'il existe physiquement des stocks. Qu'une récolte soit déficitaire n'implique absolument pas une absence totale de produits à vendre.

- Dans tous les cas envisagés, il faut, pour accéder aux denrées alimentaires, pouvoir aussi accéder à un marché, ce qui est impossible si les prix sont trop élevés.

- Il existe des titres, des droits d'entrée, qu'il s'agisse de la récolte à venir ou d'argent versé comptant. Les véritables difficultés apparaissent quand il n'y a plus de moyens de paiement, quelle qu'en soit la forme, pour se procurer une denrée qui existe effectivement. Cela signifie que les problèmes sont au moins autant des problèmes d'échange et de marché que des problèmes de production.

Ce mécanisme-là est compris, instinctivement du moins, et est intégré. Il fait partie des savoirs tacites que se partagent les agents économiques et que, d'une certaine façon, les évêques sont capables d'exposer et de formaliser. Les mesures prises servent à éviter les stockages abusifs, l'accaparement, bref les comportements relevant de l'avaritia. Il faut en effet que les biens circulent, ce qui est, in fine, la définition de l'usage chrétien de la richesse. Les maxima des prix fixés par Charlemagne sont là pour maintenir active cette circulation des 
biens matériels qui apparaît comme le reflet des circulations immatérielles existant entre la terre et l'au-delà.

D'autres textes, parfois plus tardifs éclairent encore mieux ces pratiques et ces savoirs. En 1124, par exemple, à Bruges, lors de la grande famine qui ravagea la Flandre, le comte prit, en plus des dispositions caritatives habituelles de distribution de nourriture, une série de mesures intervenant directement sur le fonctionnement du marché. Outre une réglementation concernant la production, il fit d'une part baisser le poids des pains et d'autre part distribuer de petites sommes d'argent aux membres de sa familia afin qu'ils puissent s'approvisionner par achat. Les deux mesures prises ensemble ont un sens très clair : Charles le Bon fournit un droit d'entrée sur le marché à ses dépendants, en établissant une adéquation entre le prix des choses et les quantités d'argent disponibles par les consommateurs ${ }^{84}$.

\section{La diversité des scènes sociales et l'échange marchand}

Les choses ont une valeur et il est souvent possible de la déterminer, qu'il s'agisse de terres, de quantités de blés ou encore d'objets produits par l'artisanat monastique.

Cette valeur n'est cependant pas une donnée absolument stable et dépend pour beaucoup de ce que recherchent les acteurs ou de ce qu'ils ont besoin de faire au moment où ils procèdent à l'estimation ou à l'échange. L'abbé bénédictin connaît les règles de formation des prix et sait, en tout état de cause, où il faut aller chercher l'information. Cela ne l'empêche pas, cependant, d'intervenir, en fixant des prix, alors que ceux-ci se sont formés en dehors de lui. Il existe en effet plusieurs déterminants à prendre en considération, dont le moindre n'est pas le salut de l'âme de l'acteur. S'il existe donc des mécanismes et des automatismes, liés à des procédures et à des règles fixes, il existe aussi une diversité de motivations. Les comportements ne peuvent pas se ramener à l'unité : les scènes sociales sont diverses et il faut agir parfois simultanément de manière différenciée, en fonction de contraintes propres à chacune d'entre elles auxquelles répondent des règles particulières. Le même acte, apparemment simple, acheter ou vendre, prend des formes et entraîne des conséquences qui ne sont pas toujours les mêmes. Vendre son patrimoine pour préparer son départ, ce n'est pas la même chose que se séparer d'une parcelle pour se procurer un animal ou une arme. Dans

84 - Jeff RIDER (éd.), Vita Karoli comitis Flandrie et vita domni Ioannis Morinensis episcopi, Turnhout, Brepols, 2006, p. 38, chap. 12 ; Id., Galbertus notarius brugensis. De multro, traditione et occisione gloriosi Karoli comitis Flandriarum, Turnhout, Brepols, 1994, p. 78-80. 
ces conditions, le bien faisant l'objet d'une transaction n'est pas estimé de la même manière, puisque dans un cas il faut que le vendeur en tire le prix le plus élevé possible et que, dans l'autre, la contre-valeur est un point secondaire, l'essentiel résidant dans l'acquisition d'un objet qui rend possible la continuation de l'exploitation ou le maintien du statut social. De même, les transactions foncières préparant un mariage obéissent à des règles propres qui dérogent à la loi du marché. Mais il y a des règles et il y a un marché par rapport à quoi les agents se situent : ils savent ce qu'ils font et agissent en toute conscience en fonction des buts qu'ils se sont assignés et qu'ils poursuivent. Le marché fournit des références, il ne crée pas d'obligation ou, si l'on préfère, n'induit pas des comportements automatiques. En particulier, le prix du marché n'est en aucune manière considéré comme un prix juste, puisque d'une part les abbés ont l'obligation de le considérer comme surévalué et que, d'autre part, ils les manipulent selon des critères qui leur sont propres afin, précisément, de s'approcher d'une évaluation qui ait à voir davantage avec la justice qu'avec la raison. Nous avons focalisé le propos sur l'abbé estimateur et expert : il n'est pas le seul, il s'en faut, à avoir la possibilité de fixer des prix à un niveau différent du prix formé sur le marché. Le souverain, tout d'abord, s'arroge le droit de le faire et le fait à chaque fois qu'il estime nécessaire de le faire, parce que, par exemple, la famine frappe son peuple. Il y a là une constante dans les comportements médiévaux que l'on retrouve aussi bien chez Charlemagne que chez le comte de Flandre Charles le Bon ou, beaucoup plus tard, chez les officiers florentins qui pourvoient à l'annone ${ }^{85}$. Les causes de leur action sont bien sûr formulées différemment, mais toujours se retrouvent le désir de stabilité sociale et d'ordre, la peur des troubles que la famine, ressentie comme une injustice, pourrait faire naître, et le désir de plaire à Dieu, aussi bien pour assurer son propre salut dans l'au-delà que la sauvegarde de la société ici-bas. On a donc un modèle où certains, qui détiennent le pouvoir ou disposent d'une autorité morale, savent ce qu'il faut faire pour les autres et sont en mesure d'imposer leurs décisions.

Il en va de même, mutatis mutandis, des notables désignés pour servir d'experts dans les commissions chargées de mesurer la valeur des biens des orphelins et les conseiller pour les protéger dans les temps de misère. Leur rapport aux prix de marché est modifié par la nécessité dans laquelle ils se trouvent d'avoir à agir de façon qui soit moralement recevable, c'est-à-dire de donner des estimations et de prendre des décisions qui permettent aux

\footnotetext{
${ }^{85}$ - Voir le texte fameux sur la famine de 1329-1330 à Florence de Giovanni VILlANI, Nuova Cronica, éd. par G. Porta, Parme, Guanda, 1990, vol. 2., liv. 11, chap. 119.
} 
orphelins de survivre ${ }^{86}$. L'évaluation obéit alors à des règles d'équité qui ne sont pas celles que les procédures du marché admettent ou promeuvent. Cependant, si les agents en position d'autorité ont une large marge de manœuvre, il n'en va pas de même pour les petits et les faibles, qui doivent subir ces «prix justes», ou ces décisions équitables, sans avoir la possibilité d'intervenir dans les décisions qui les forment.

Les marchés, compris comme étant des lieux d'échange, apparaissent ainsi comme des instruments de mise en œuvre des procédures d'évaluation. Celles-ci sont bien maîtrisées par les acteurs, dès le $\mathrm{IX}^{\mathrm{e}}$ siècle au moins. Cet instrument est cependant manipulé par les agents en fonction d'impératifs moraux, sociaux ou politiques : il n'est pas suffisant pour assurer le fonctionnement des différents types d'échanges existant dans les sociétés du haut Moyen Âge. Le prix est le point d'application d'interventions qui permettent d'utiliser les choses non seulement pour obtenir son salut mais aussi pour assurer la continuité de la lignée ou encore la stabilité de l'ordre social. Ces différents buts induisent des formes diversifiées d'échange qui coexistent mais au sein desquelles l'échange marchand occupe une place essentielle, parce qu'il permet l'établissement de points de référence qui donnent sens aux échanges non marchands. Par-delà ces questions, toutefois, l'essentiel est d'admettre que les acteurs savent, dans la plupart des cas, ce qu'ils font et quel genre d'échange, marchand ou non marchand, commercial ou cérémoniel, ils pratiquent en fonction des différentes scènes sociales sur lesquelles ils se trouvent au moment de l'action ${ }^{87}$. L'idéal de justice et la position morale revendiquée et assumée par les acteurs dominants de la scène sociale ne doivent cependant pas masquer qu'il existe aussi des acteurs s'efforçant de profiter de toutes les opportunités, les spéculateurs auxquels s'attaque le concile de 829, par exemple, ou tous ceux qui, achetant les récoltes sur pied, contribuent à l'appauvrissement des paysans les plus faibles. Ceux-là sont menacés doublement, et par l'action des détenteurs d'argent et de réserves alimentaires et par celle des hommes ayant autorité dont l'action à finalité apparemment morale peut cependant parfaitement s'accommoder de la recherche du profit et de celle de positions monopolistiques, tout aussi nuisibles, à terme, à des acteurs affaiblis ou appauvris.

Laurent Feller

\footnotetext{
86 - Pere Benito, «Marché foncier et besoin d'expertise dans la Catalogne du $\mathrm{X}^{\mathrm{e}}$-XII ${ }^{\mathrm{e}}$ siècles. Le rôle des boni homines comme estimateurs de biens ", in C. DENJEAN et L. FELleR (dir.), Expertise et valeur des choses au Moyen Âge, sous presse.

87 - Voir sur ces points Alain Testart, «Échange marchand, échange non marchand», Revue française de sociologie, 42-4, 2001, p. 719-748.
} 
Université Paris 1 Panthéon-Sorbonne/Institut Universitaire de France UMR 8589/LAMOP 